\title{
The outskirts of Cygnus OB2 ${ }^{\star} \star \star \star$
}

\author{
F. Comerón ${ }^{1, \star \star \star}$, A. Pasquali ${ }^{2}$, F. Figueras ${ }^{3}$, and J. Torra ${ }^{3}$ \\ ${ }^{1}$ European Southern Observatory, Karl-Schwarzschild-Strasse 2, 85748 Garching, Germany \\ e-mail: fcomeron@eso.org \\ 2 Max-Planck-Institut für Astronomie, Königstuhl 17, 69117 Heidelberg, Germany \\ e-mail: pasquali@mpia-hd.mpg.de \\ 3 Departament d'Astronomia i Meteorologia, Universitat de Barcelona, 08028 Barcelona, Spain \\ e-mail: [jordi; cesca]@am.ub.es
}

Received 7 April 2008 / Accepted 15 May 2008

\section{ABSTRACT}

Context. Cygnus OB2 is one of the richest OB associations in the local Galaxy, and is located in a vast complex containing several other associations, clusters, molecular clouds, and HII regions. However, the stellar content of Cygnus OB2 and its surroundings remains rather poorly known largely due to the considerable reddening in its direction at visible wavelength.

Aims. We investigate the possible existence of an extended halo of early-type stars around Cygnus OB2, which is hinted at by nearinfrared color-color diagrams, and its relationship to Cygnus OB2 itself, as well as to the nearby association Cygnus OB9 and to the star forming regions in the Cygnus X North complex.

Methods. Candidate selection is made with photometry in the 2MASS all-sky point source catalog. The early-type nature of the selected candidates is confirmed or discarded through our infrared spectroscopy at low resolution. In addition, spectral classifications in the visible are presented for many lightly-reddened stars.

Results. A total of 96 early-type stars are identified in the targeted region, which amounts to nearly half of the observed sample. Most of them have featureless near-infrared spectra as expected from OB stars at the available resolution. Another 18 stars that display Brackett emission lines can be divided between evolved massive stars (most likely Be stars) and Herbig Ae/Be stars based on their infrared excesses. A component associated with Cygnus OB9/NGC 6910 is clearly identified, as well as an enhancement in the surface density of early-type stars at Cygnus X North. We also find a field population, consisting largely of early B giants and supergiants, which is probably the same as identified in recent studies of the inner $1^{\circ}$ circle around Cygnus OB2. The age and large extension of this population discards a direct relationship with Cygnus OB2 or any other particular association.

Conclusions. Earlier claims of the possible large extent of Cygnus OB2 beyond its central, very massive aggregate seem to be dismissed by our findings. The existence of a nearly ubiquitous population of evolved stars with massive precursors suggests a massive star formation history in Cygnus having started long before the formation of the currently observed OB associations in the region.

Key words. stars: early-type - Galaxy: open clusters and associations: individual: Cygnus OB2 -

Galaxy: open clusters and associations: individual: Cygnus OB9

\section{Introduction}

Cygnus OB2 is one of the richest OB associations known in our Galaxy, and it is the most nearby one of its kind at less than $2 \mathrm{kpc}$ from the Sun (Knödlseder 2003). It is also a unique resource for the observational study of the upper end of the stellar mass function (e.g., Herrero et al. 1999): O stars, Wolf-Rayet (WR) stars, Luminous Blue Variables (LBVs), B[e] stars... are all classes with representatives in Cygnus OB2. This association thus provides, in a single complex, the most complete and accessible showcase of the variety found among the hottest and most massive stars.

$U B V$ photometry reveals the presence of hundreds of early-type, heavily-obscured stars in Cygnus OB2

* Based on observations collected at the Centro Astronómico HispanoAlemán (CAHA) at Calar Alto, operated jointly by the Max-Planck Institut für Astronomie and the Instituto de Astrofísica de Andalucía (CSIC).

$\star \star$ Tables $1-9$ are only available in electronic form at http: //www . aanda.org

$\star \star \star$ Visiting astronomer at the Vatican Observatory.
(Massey \& Thompson 1991; Kiminki et al. 2007). However, a more real measure of its richness is given by star counts in the near infrared, where the large, patchy foreground extinction in the direction of the association is greatly reduced. Using this technique, Knödlseder (2000) has inferred a content of 2600 OB stars, including over 100 O-type stars and evolved stars having massive progenitors. Support for this estimate has been provided by Comerón et al. (2002) using near-infrared low resolution spectroscopy. High signal-to-noise ratio spectra in the visible of the least obscured objects of this sample carried out by Hanson (2003) has confirmed the classification of the vast majority of the early-type star candidates listed by Comerón et al. (2002). However, it has also shown that a large fraction of them are giant and supergiant B stars rather than $\mathrm{O}$ stars. This suggests a picture more complex than that of a single, recent starburst less than $3 \mathrm{Myr}$ ago giving rise to the association (Massey \& Thompson 1991). It also casts doubts on the actual membership of these stars in Cygnus OB2, given their older ages and their extended spatial distribution when compared to the strongly-clustered, earliest-type stars at the center of the association. Since the precursors of the extended population 
dominated by early B-type giants and supergiants must have been O-type stars, the region must have been producing massive stars long before the formation of the major young clusters and associations currently observed there.

We investigate the nature of this population and its possible relationship with Cygnus OB2 by studying the early-type stellar content in a region lying between $1^{\circ}$ an $2^{\circ}$ from the center of the association. Earlier results of this work have already revealed the existence of interesting objects possibly related to Cygnus OB2 well beyond the boundaries of the central cluster. Examples are WR 142a, a WC8 Wolf-Rayet star about $1.3^{\circ}$ from the center of Cygnus OB2 (Pasquali et al. 2002), and the very massive O4If runaway star $\mathrm{BD}+43^{\circ} 3654$ more than two degrees away (Comerón \& Pasquali 2007). The study of the outskirts of Cygnus OB2 is also motivated by its overlap with other structures tracing recent star formation, most notably the Cygnus OB9 association and several compact HII regions and embedded clusters belonging to the Cygnus X molecular cloud complex (Schneider et al. 2006).

We use the same technique based on 2MASS JHK colors used in Comerón et al. (2002) to identify OB stars in the targeted region. Infrared spectroscopy allows us to identify new young intermediate-mass stars and evolved massive stars, both characterized by their emission in $\mathrm{Br} \gamma$, as well as other likely early-type stars without noticeable emission lines. We also provide spectral types of many of these objects whose relatively light obscuration makes them accessible to spectral classification in the blue. This paper can thus be regarded as a continuation of the previous work of Comerón et al. (2002) \& Hanson (2003), now probing the largely uncharted region beyond the limits of Cygnus OB2 explored thus far. The reader is referred to the recent review by Reipurth \& Schneider (2008) for a detailed description of the current state of knowledge of the vast extent of Cygnus star forming complexes, and, in particular, of the Cygnus OB2 association and the Cygnus $\mathrm{X}$ region.

\section{Target selection}

The use of the $(J-H),\left(H-K_{\mathrm{S}}\right)$ diagram to distinguish distant early-type stars from late-type evolved stars is founded on the difference in intrinsic colors between both populations. The interstellar reddening law keeps the loci occupied by bright, reddened early- and late-type stars separated by a gap of $\sim 0.4$ mag in $(J-H)$ at any given $\left(H-K_{\mathrm{S}}\right)$ color, in the sense of the $(J-H)$ color being bluer; see, e.g., Comerón \& Pasquali (2005) for an application of this technique to the identification of the star responsible for the ionization of the North America and Pelican nebulae. The location of a star along the band defined by the reddened colors of early-type stars obscured by different amounts is not a proof of its early spectral type, since a certain degree of contamination of this locus is caused by evolved stars with particular photometric properties. Nevertheless, the signature of a rich association such as Cygnus OB2 in the $(J-H),\left(H-K_{\mathrm{S}}\right)$ diagram is clearly indicated by the presence of an enhancement of the population of this locus (see, e.g., Fig. 2 of Comerón et al. 2002). This makes the early-type population easily identifiable even in regions where its areal density is far below that of cool giants, thus extending the sensitivity threshold to the earlytype population of the association well beyond the $\simeq 1^{\circ}$ radius where it stands out in infrared starcounts.

The presence of early-type stars beyond $1^{\circ}$ from the center of Cygnus OB2 is clearly indicated in Fig. 1, where we plot the 2MASS $(J-H),\left(H-K_{\mathrm{S}}\right)$ diagram of point sources located between $1^{\circ}$ and $2^{\circ}$ from the approximate center of Cygnus OB2

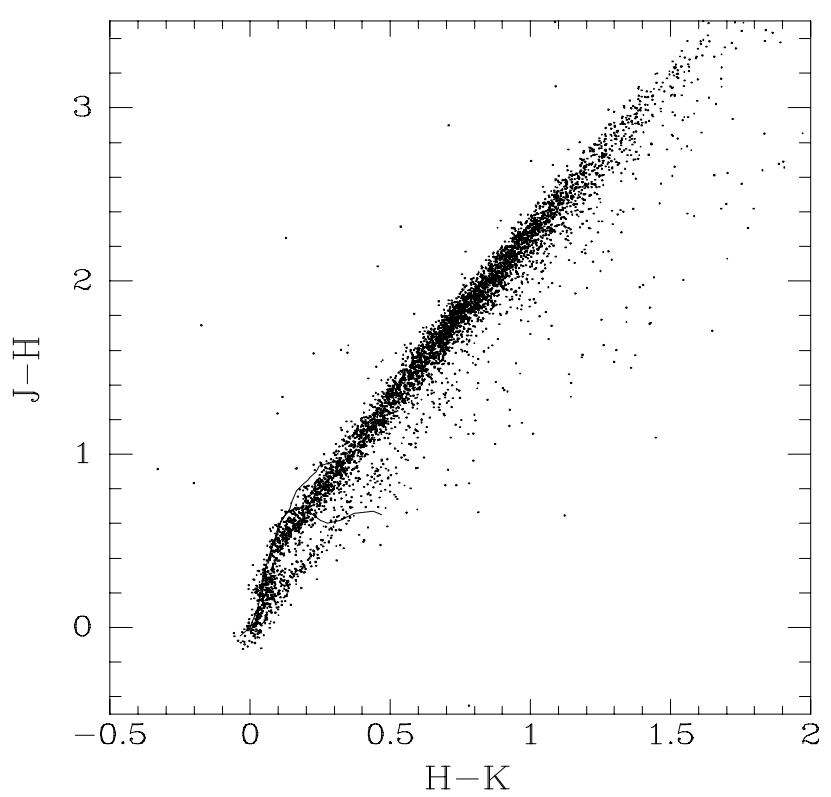

Fig. 1. $(J-H),(H-K)$ diagram of the field surrounding Cygnus OB2 between $1^{\circ}$ and $2^{\circ}$ from the approximate center of the association, at $\alpha(2000)=20^{\mathrm{h}} 32^{\mathrm{m}} 26^{\mathrm{s}}, \delta(2000)=+40^{\circ} 52^{\prime}$. The plotted magnitudes are from the 2MASS catalog. Only objects brighter than $K_{\mathrm{S}}=10$ are included. Candidate early-type stars are located along a band that has its origin near $(J-H)=0,(H-K)=0$ and traces the reddening vector.

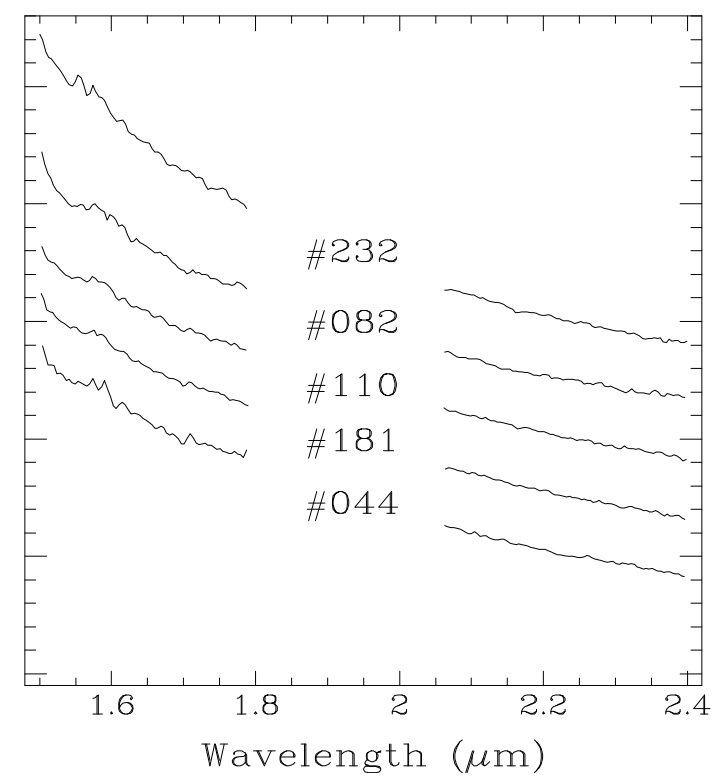

Fig. 2. Typical examples of suspected reddened, early-type stars. These stars appear featureless at the resolution and signal-to-noise ratio of our near-infrared spectra.

at $\alpha(2000)=20^{\mathrm{h}} 32^{\mathrm{m}} 26^{\mathrm{s}}, \delta(2000)=+40^{\circ} 52^{\prime}$. A band of objects with varying reddening runs along the locus of early-type stars, well separated from the dominant band defined by cool background stars, which runs above it. It is interesting to note that the number of candidate $\mathrm{OB}$ stars in the region between $1^{\circ}$ and $2^{\circ}$ from the center of Cygnus OB2 is comparable to that found within $1^{\circ}$ from the center. In addition, the spread in extinctions is larger than in the central region, indicating that many OB stars may be very obscured and thus inaccessible to survey in the visible. 
To confirm the nature of individual stars lying in the earlytype locus described above and their likely membership in the association, we obtained low-resolution near-infrared spectroscopy of 232 stars in the region between $1^{\circ}$ and $2^{\circ}$ from the center of the association. The selected stars have $(J-H)-$ $1.70\left(H-K_{\mathrm{S}}\right)<0.20$, implying a closer position to the locus of early-type stars than to that of late-type stars; and $K_{\mathrm{S}}<8.8$, allowing us to obtain, with a reasonable investment of observing time, a large number of $K$-band spectra. We also obtained classification-quality spectra in the blue of 30 of the stars with near-infrared spectra consistent with early types and membership in the association, and whose foreground obscuration was relatively low as judged from their $B$ magnitude listed in the USNO-B catalog (Monet et al. 2003).

We adopt here the rather loose denomination "Cygnus OB2 halo members" to refer to early-type stars and young stellar objects located in the area under discussion, and suspected to lie at the same distance as the association. This is not meant to indicate confirmed membership in the Cygnus OB2 association. Moreover, some of the stars we discuss are actually located within the boundaries of Cygnus OB9 (including some members of the open cluster NGC 6910 belonging to that association) or are associated with star forming regions in Cygnus $\mathrm{X}$ like DR 17 or DR 21 that are normally not considered to be a part of Cygnus OB2.

Hanson (2003) has produced a detailed discussion on the distance to Cygnus OB2 based on current available calibrations of the intrinsic properties of upper main sequence stars. Following these conclusions, we adopt a distance of $1.45 \mathrm{kpc}$ (distance modulus 10.80 ) to the region. We also assume that all the structures discussed here belong to a physically-coherent region lying at approximately that distance, rather than belonging to disconnected entities along the line of sight, as further discussed in Sect. 4.4.

\section{Observations}

\subsection{Near-infrared spectroscopy}

We carried out near-infrared spectroscopic observations during two observing runs at the Calar Alto observatory. The first one took place from 16 June to 1 July 2002 at the $1.23 \mathrm{~m}$ telescope, and the second one from 31 July to 2 August 2004 at the $2.2 \mathrm{~m}$ telescope. The instrument used was in both cases MAGIC, a NICMOS3-based near-infrared camera and spectrograph. We obtained the spectra using the resin-replica grism providing a resolution $\lambda / \Delta \lambda=240$ over the $1.50-2.40 \mu \mathrm{m}$ range with the $1^{\prime \prime}$ slit used. Each star was observed at six positions along the slit, with exposure times per position ranging from 20 s (stacking 10 integrations of $2 \mathrm{~s}$ ) for the brightest stars to $60 \mathrm{~s}$ (stacking 20 integrations of $3 \mathrm{~s}$ ) for the faintest, with exposure times determined by the 2MASS $K_{\mathrm{S}}$ magnitude and the aperture of the telescope used. We carried out the extraction and calibration of the spectra with dedicated IRAF ${ }^{1}$ scripts. The frames obtained at consecutive slit positions were subtracted from each other to cancel out the sky contribution to the spectrum, and were divided by a flat field frame. A one-dimensional object spectrum was then extracted from each sky-subtracted, flat-fielded frame. We performed the wavelength calibration of each individual spectrum with the $\mathrm{OH}$ airglow lines in each frame as

\footnotetext{
1 IRAF is distributed by NOAO, which is operated by the Association of Universities for Research in Astronomy, Inc., under contract to the National Science Foundation.
}

a reference (Oliva \& Origlia 1992). The wavelength-calibrated spectra extracted at each sky position were then coadded, with deviant pixels due to detector defects or cosmic ray hits automatically clipped off. Cancellation of telluric features was achieved by ratioing the object spectra by those of the nearby G5IV star HD 190771, which is expected to be featureless at the resolution employed, reduced in the same manner. Finally, we performed relative flux calibration by multiplying the reduced spectra by that of a $5700 \mathrm{~K}$ blackbody, which should be a good approximation to the spectral energy distribution of an unreddened G5IV star in the wavelength range covered by our spectra.

\subsection{Visible spectroscopy}

Spectroscopy in the visible of 26 targets suspected to be reddened early-type stars from their near-infrared photometric and spectroscopic properties was obtained at Calar Alto using CAFOS, the facility visible-light imager and low-resolution spectrograph, in an observing run between 1 and 12 July 2005. We obtained spectra of four additional stars with an identical instrumental setup at the same telescope in an observing run between 17 and 25 August 2006. The grism used covered the range shortwards of $\lambda=6350 \AA$ at a resolution of $\lambda / \Delta \lambda=1000$ and with a $1^{\prime \prime} 5$ slit. We based the exposure times on the $B$ magnitude listed in the USNO-B catalog, and ranged from $10 \mathrm{~min}$ to $180 \mathrm{~min}$. For integration times longer than $30 \mathrm{~min}$, the exposures were split in blocks of $30 \mathrm{~min}$, and the individual extracted and reduced spectra were then stacked together. Spectra of three lamps of $\mathrm{HgCd}, \mathrm{He}$, and $\mathrm{Rb}$ were taken between subsequent exposures for wavelength calibration, to minimize the effects of instrument flexure. The frames containing the raw spectra were subtracted from bias and divided by a spectroscopic flat field, and the spectra were subsequently extracted from each one of them. The individual wavelength-calibrated spectra were coadded after identification and removal of cosmic ray hits. The coadded spectra were then normalized to the interpolated continuum, to facilitate feature recognition and comparison to spectral atlases of these generally heavily-reddened objects.

\section{Results}

\subsection{Infrared spectral classification and membership}

Despite the limited resolution and signal-to-noise ratio of our infrared spectra, it is possible to broadly classify our entire sample of stars into eight classes:

- Reddened stars with featureless spectra: the absence of spectral features at the resolution and signal-to-noise ratio of our spectra is expected from hot stars earlier than mid-B type (Hanson et al. 1996), at which the Brackett lines begin to be clearly visible. Their significant foreground reddening and the relatively bright limiting magnitude of our sample ensures that these are luminous early-type stars. Whereas G-type stars would also appear featureless in our spectra, their infrared colors would in general exclude them from our sample (Sect. 2), and main sequence G stars would be too faint at the distances where reddening begins being noticeable. The sample of reddened stars with featureless spectra (see examples in Fig. 2) is thus expected to have little or no contamination by objects other than $\mathrm{O}$ and $\mathrm{B}$ stars, as confirmed by our visible spectroscopy of some of the least heavily-reddened stars in this category. A similar conclusion 
was reached by Hanson (2003), who obtained visible spectroscopy of 14 out of the 31 stars selected in the same manner by Comerón et al. (2002), and confirmed that all of them are indeed early-type stars. Given the rarity of such objects in the field, we consider that this class provides a largely uncontaminated sample of members of the Cygnus region. This is the class with most members in our sample, and the 66 stars belonging to it are listed in Table 1. Visible spectral classifications exist for 28 of these stars, of which 24 are presented in this work for the first time.

- Reddened stars without measurable absorption lines, but with emission lines (particularly Bry): stars in this category are expected to belong to two distinct, unrelated classes. The first one is composed of evolved massive stars such as $\mathrm{B}[\mathrm{e}]$, Of, LBV stars or, more likely, early Be stars (Morris et al. 1996), all of which have massive precursors. The second class is composed of intermediate-mass Herbig Ae/Be stars. We consider both classes of objects as possible members of the Cygnus complex as well, and we discuss in Sect. 4.2 how we can distinguish between both. We list in Table 2 the 19 stars in this category, whose spectra are plotted in Fig. 3. Visible spectra are available for 5 of them, and a sixth one, the Wolf-Rayet star WR 142a, has been classified as WC8 by Pasquali et al. (2002) from its infrared spectrum. Six of those stars, including WR 142a, had been previously recognized in objective prism surveys as emission-line stars.

- Lightly or no reddened stars with featureless spectra: a total of 31 stars in our sample show featureless infrared spectra, but their colors indicate little reddening in their direction, thus casting doubts as to their membership in the Cygnus complex. Fortunately, spectral classifications in the visible, as well as photometric classifications based on Vilnius photometry, are available for most of these stars, showing that the sample can be split into two groups:

- O and early B stars with light reddening, whose apparent magnitudes are consistent with the adopted distance modulus of Cygnus OB2. We consider these stars as likely members of the Cygnus complex, placed in the least reddened parts of the region, and we list them in Table 3. We have included in it star \#30, classified as B8 on the basis of its Vilnius photometry. Such spectral type is unlikely, however, as a B8 star should show noticeable Brackett-series lines in our near-infrared spectra. An earlier B-type seems more likely, and we thus consider it as a possible member of the complex, although with an uncertain spectral type;

- virtually unreddened F-G stars, some of which have high proper motions. We consider those as non-members and we list them in Table 4.

Only 11 stars in this class, mostly faint, have not been observed spectroscopically and it is not possible to assign them to either of those two subclasses. To preserve our sample of Cygnus complex members as uncontaminated as possible, we list these stars separately within Table 4 and we do not consider them among the possible members.

- Stars with obvious Bry absorption, and possibly other Brackett lines as well: these are unambiguously classified as late-B, A or early-F stars. Their infrared colors generally indicate little or no foreground extinction, suggesting distances closer than those of the members of Cygnus OB2. With very few exceptions, spectral classifications in the visible exist in the literature for most of these stars, confirming the above range of spectral types. We thus consider the 48 lightly-reddened objects that we identify in this category,

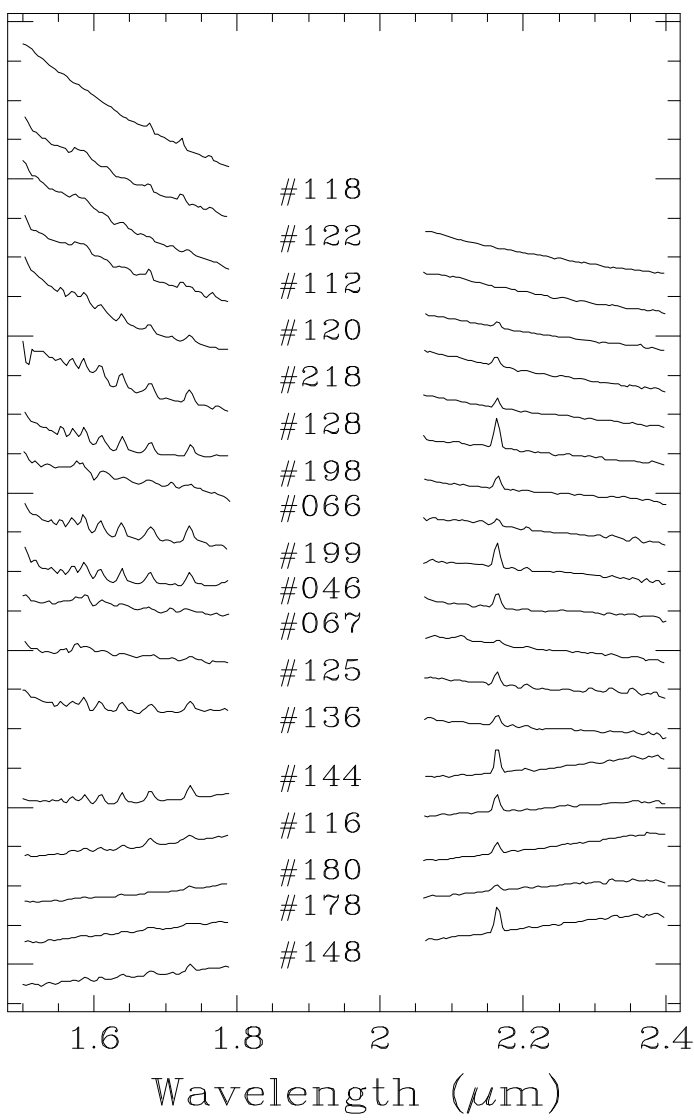

Fig. 3. Near-infrared spectra of the stars in our sample that display Brackett-series lines in emission. For convenience, the spectra are sorted by increasing reddening from top to bottom. The spectrum of an additional emission-line star, the Wolf-Rayet WR 142a, can be found in Pasquali et al. (2002). The sample shown here is expected to contain representatives of two different classes, classical Be stars and intermediate-mass Herbig Ae/Be stars, with the reddest stars belonging to the latter category.

listed in Table 5, as foreground stars. A few exceptions are found: 4 stars have clearly reddened colors and still display obvious Brackett absorption lines indicating spectral types in the late-B to early-F range. Since their apparent magnitudes and reddening indicate an intrinsically high luminosity, these objects are probably giants or supergiants. Their possible membership in the Cygnus region is unclear, and we will not include them in our census of possible members. We list them separately in Table 5 and display some typical spectra in Fig. 4.

- Red giant branch stars: although the infrared colors of these stars normally place them on the locus of reddened late-type stars, infrared excesses at $2 \mu \mathrm{m}$ can move them to the region of interest of the $(J-H),\left(H-K_{\mathrm{S}}\right)$ diagram. This seems to be the case of most of the 16 stars in our sample that clearly display $C O$ bands starting at $2.29 \mu \mathrm{m}$ despite having been color-selected. They are listed in Table 6, and display a wide range of foreground reddening, as is obvious in the representative examples shown in Fig. 4. We note, however, that one member of this class, star \#221, is projected on the DR 21 star forming region, appears extended at $2.2 \mu \mathrm{m}$ and displays a spectral energy distribution at longer wavelengths (Davis et al. 2007) characteristic of the Class I/Class II transition (Adams et al. 1987). It is thus likely to be a young stellar object belonging to DR 21, and still surrounded by 

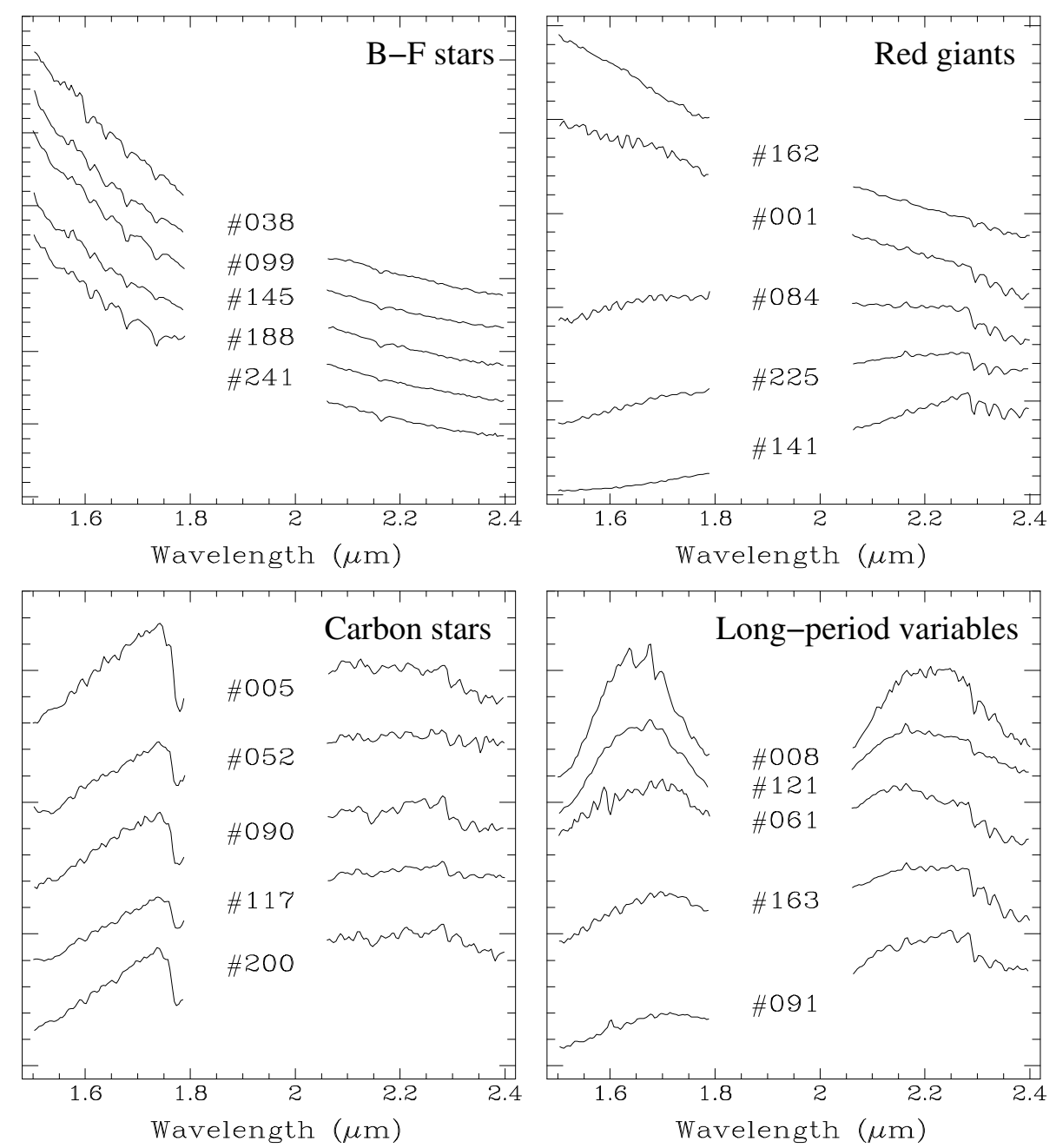

Fig. 4. Typical near-infrared spectra of the different classes of stars contaminating our sample of color-selected, early-type candidate members of the Cygnus OB2 region, which are described in Sect. 4.1.

substantial amounts of gas and dust with its $\mathrm{CO}$ absorption being produced in its circumstellar envelope. At the available resolution and signal-to-noise ratio its spectrum (Fig. 5) is typical of a cold photosphere, except perhaps for its broader $C O$ bands.

- Carbon stars: their frequent infrared excesses also place carbon stars in the region of the $(J-H),\left(H-K_{\mathrm{S}}\right)$ diagram occupied by early-type stars. They are easily recognized by the numerous features in the $K$ window, in addition to the $\mathrm{CO}$ bands, and, most particularly, by the prominent $\mathrm{C}_{2}$ band at $1.77 \mu \mathrm{m}$ just below the telluric $\mathrm{H}_{2} \mathrm{O}$ cutoff (Lançon \& Wood 2000, Fig. 4). We find 29 such stars in our sample, which are listed in Table 7. Only the two brightest ones in $K_{\mathrm{S}}$ had been previously recognized as carbon stars, and most of the others are IRAS point sources. We also note that one of the stars that we classify as a red giant in Table 6, star \#9, has been identified as a carbon star by Chen \& Chen (2003), although we do not identify its characteristic features in the near-infrared.

- Long-period variables: the infrared colors of Mira and semiregular long-period variables makes them another potential contaminant of the early-type locus. The 14 members of this class that we identify in our sample (Table 8, Fig. 4) are easily distinguished by their prominent $\mathrm{CO}$ bands as well as by the marked broad absorption due to $\mathrm{H}_{2} \mathrm{O}$ in

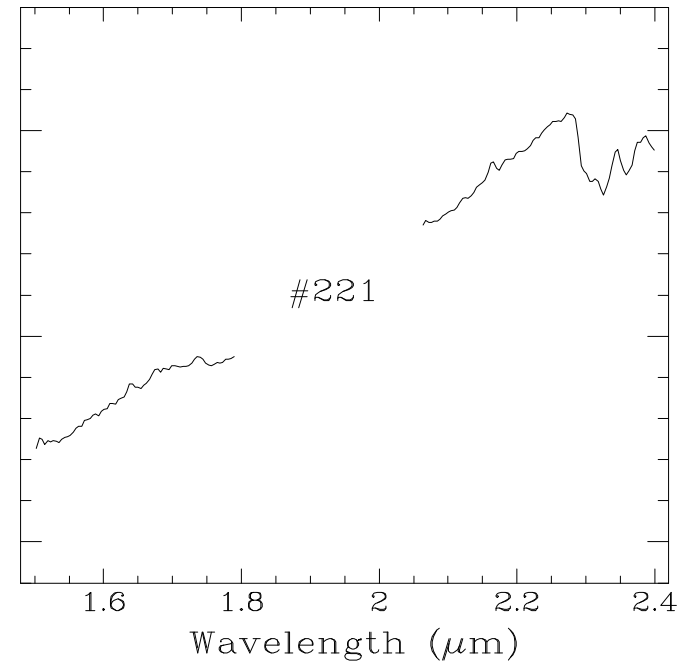

Fig. 5. Spectrum of the likely young stellar object \#221 in the DR 21 star forming region.

their extended envelopes that dominates the spectral energy distribution in the region above and below the telluric feature separating the $H$ and $K$ bands. 


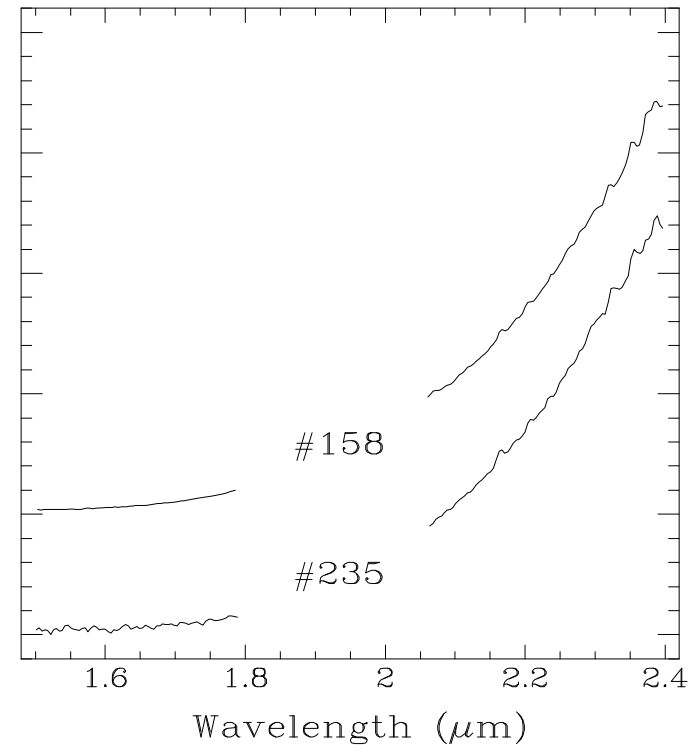

Fig. 6. Spectra of the extremely red sources \#158 and \#235. Star \#158 is the well-studied massive protostellar object AFGL 2591 (see text). Note the similar appearance of the weak Br $\gamma$ emission line at $2.166 \mu \mathrm{m}$.

- Protostellar sources: two objects in our sample display extremely red colors $\left(H-K_{\mathrm{S}}=2.76\right.$ and 4.28, respectively) and featureless spectra, except for hints of $\operatorname{Br} \gamma$ emission (Table 9). The first one is identified as the well-known source AFGL 2591, a high-mass protostellar object that has been the subject of numerous studies; see, e.g., Benz et al. (2007), Poelman \& van der Tak (2007), as well as the review by Reipurth \& Schneider (2008) for recent discussions on this source. The second one is identified as IRAS 20249+3953, for which no detailed observations have been published thus far. The obvious similarity of its near-infrared spectrum to that of AFGL 2591, as seen in Fig. 6, leads us to classify it as a massive protostar also, perhaps in an earlier evolutionary stage than AFGL 2591, given its much redder colors. The source IRAS 20249+3953 was undetected in the search for $\mathrm{SiO}$ maser emission by Nakashima \& Deguchi (2003).

\subsection{Infrared color-color diagram}

The distribution of objects of our sample in the near-infrared color-color diagram is presented in Fig. 7. The most abundant class of stars, those displaying featureless near-infrared spectra, are well aligned along the reddening vector, displaying the colors of reddened normal early-type photospheres without indications of near-infrared excess. Based on the position of the reddest stars with the colors and near-infrared spectra of normal photospheres, we estimate a maximum reddening toward the halo of Cygnus $\mathrm{OB} 2$ of $A_{V_{\max }} \simeq 15 \mathrm{mag}$, or $A_{K_{\max }} \simeq 1.7 \mathrm{mag}$.

The bluest emission-line stars tend to appear slightly to the right of the limiting reddening vector having its origin at the position of the earliest unreddened stars, thus indicating the existence of moderate amounts of circumstellar excess. However, a break is easily identified at $(H-K)>0.8$ as emission-line stars with redder colors are all far more removed from the limiting reddening vector. We interpret this as a consequence of the existence of two classes of emission-line stars in our sample, as discussed in Sect. 3.1. Classical Be stars have infrared excesses produced by free-free and free-bound transitions in the ionized

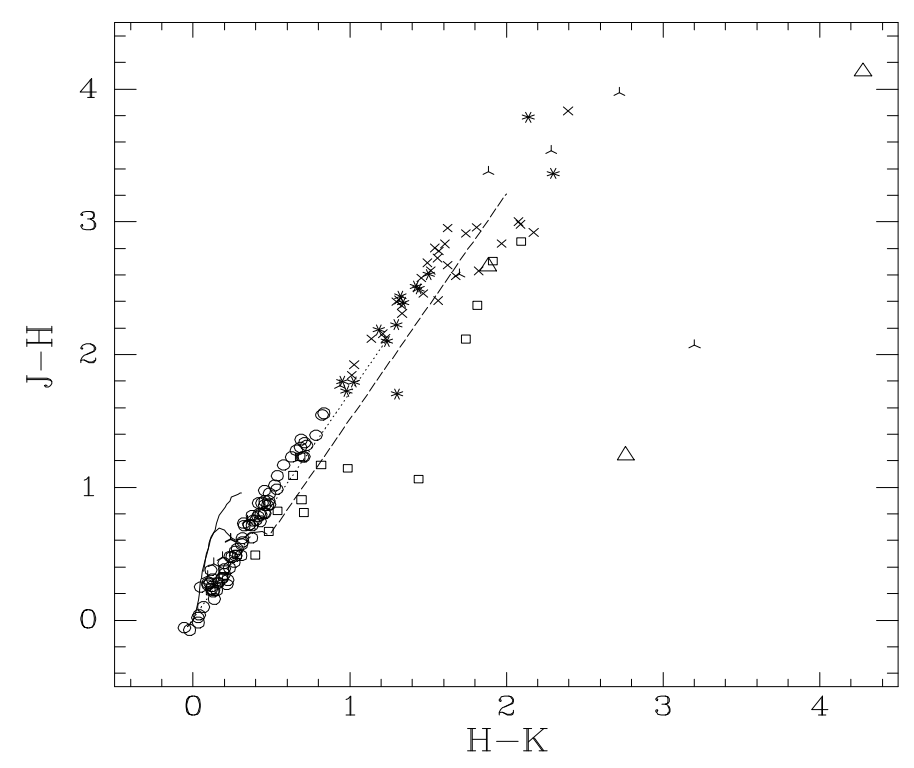

Fig. 7. Color-color diagram of all the objects in our sample, with the exception of foreground stars. Circles: stars with featureless spectra; squares: emission-line stars; triangles: massive young stellar objects; 3-pointed asterisks: red giant branch stars; 4-pointed asterisks: carbon stars; and 8-pointed asterisks: long-period variables. The length of the reddening vector (dotted line) corresponds to $20 \mathrm{mag}$ of visual extinction. The dashed line separates the loci of classical Be from Herbig Ae/Be stars.

circumstellar gas, which is thought to be distributed in a decretion disk formed as a consequence of mass loss from the atmosphere of a star rotating at nearly the break-up speed (Sigut \& Jones 2007; Ekström et al. 2008). Although the Be phenomenon may appear at all stellar ages, and also among young stars with a rapid initial rotation speed (Zorec \& Briot 1997), several observational lines of evidence indicate that it most usually results from spin-up of the star via mass transfer from a companion, near the end of the main sequence lifetime (McSwain $\&$ Gies 2007). On the other hand, Herbig Ae/Be stars are premain sequence stars that display strong infrared excesses produced by dust in their circumstellar accretion disks, particularly in the puffed-up walls delimiting their inner rims (Dullemond et al. 2001). As shown by Hernández et al. (2005), the $J H K$ color-color diagram is a useful tool for distinguishing between both classes of star and our own sample confirms the split of both classes according to their position in the $(J-H),(H-K)$ diagram, or more precisely according to the reddening-free parameter $Q=(J-H)-1.70(H-K)$ already used in Sect. 2. Classical Be star candidates cluster around $-0.18<Q<0.07$, whereas Herbig $\mathrm{Ae} / \mathrm{Be}$ candidates cover a broad range starting at $Q=-0.22$ and extending to $Q=-1.38$. The adopted dividing line between classical Be and Herbig Ae/Be stars, defined as $Q=-0.20$, is indicated in Fig. 7. Since Herbig Ae/Be fulfill our selection criteria due to their infrared excess rather than to their photospheric colors, by including them in our sample of candidate Cygnus OB2 halo members we extend it to stars less massive than the OB stars on which we focus. However, including the Herbig Ae/Be in our discussion is still useful as they are additional tracers of recent star formation.

Two of the massive young stellar objects that we include in our sample stand out in Fig. 7 due to their extreme infrared excess. Star \#158 (= AFGL 2591) is represented by the triangle at $(J-H)=1.24,(H-K)=2.76$, whereas the even more extreme star \#235 (=IRAS $20249+3953$ ) appears near the upper 
right corner of the diagram. The candidate massive young stellar object \#221 shows a modest amount of excess by comparison, occupying a position similar to that of some Herbig Ae/Be stars, which probably indicates a more evolved status. We note the apparent existence of a third object with extreme infrared colors, star \#141, which we classified among the red giant candidates on the basis of the well-defined $\mathrm{CO}$ absorption bands longwards of $2.29 \mu \mathrm{m}$ characteristic of a late-type star. However, an inspection of the 2MASS images at the position of this star clearly shows the presence of a nearby star of similar brightness at $K_{\mathrm{S}}$, which dominates at shorter wavelengths; in fact, star \#141 is not noticeable in the 2MASS J image. The strong apparent infrared excess is thus almost certainly an artifact due to contamination of the photometric measurement by the dominating bright companion.

The cool stars in our sample that we classify as non-members appear almost entirely removed from the loci occupied by the candidate members of the Cygnus OB2 halo, having in general much redder colors. This is partly caused by their intrinsically redder spectral energy distributions, but the dominant factor is their larger average distances due to their brighter infrared absolute magnitudes. Whereas we expect OB stars in Cygnus OB2 to have absolute magnitudes in the $-3<M_{K}<-5.5$ range (Martins \& Plez 2006), cool red giants are expected to reach $M_{K}=-7$ (e.g., Ferraro et al. 2000), long period variables are in the range $-6.4>M_{K}>-8.2$ (Knapp et al. 2003), and carbon stars usually have $M_{K}<-8$ (Weinberg \& Nikolaev 2001; Demers \& Battinelli 2007). They are thus easily detectable at the distance of the Perseus arm and beyond in a magnitude-limited sample like ours.

\subsection{Spectral classification in the visible}

The new spectroscopic classifications of 29 stars presented for the first time in this paper ${ }^{2}$ are listed in Col. 7 of Tables 1 and 2. Classifications were based on a comparison between our spectra and the atlas of Walborn \& Kirkpatrick (1990), which contains an abundant selection of early stars providing a very finelygrained coverage of the two-dimensional classification of the earliest spectral types, as well as an extensive discussion of the classification criteria. In the range of spectral types covered by our stars, spectral subtype classification is mainly based on the relative strengths of the HeII features with respect to HeI, most notably HeII $\lambda 4541$ vs. HeI $\lambda 4471$, for O-type stars in which HeII lines are visible; and on the SiIV/SiIII and SiII/SiIII ratios, as well as the $\mathrm{HeI} / \mathrm{H}$ ratios, for the early B stars. In turn, the luminosity class is largely derived from the appearance of the lines produced by ionized metals (CIII, SiIV) in the proximities of $\mathrm{H} \gamma$ and, most notably, the intensity of the CIII+OII feature at $4650 \AA$. Because of the large extinction in their direction, all our stars show prominent interstellar absorption features, most notably the broad band centered at $4428 \AA$ and narrower lines at $4501 \AA, 4726 \AA$, and $4762 \AA$. To estimate the uncertainty in the spectral classification, each spectrum was separately classified by each of the authors using the same reference atlas. A comparison of the individual results obtained then led us to estimate an uncertainty in each dimension of the spectral classification amounting to approximately one luminosity class and less than one spectral subtype, respectively. The spectral types listed in

\footnotetext{
${ }^{2}$ We inadvertently also observed star \#044, already classified by Hanson (2003), as noted in Table 1. For consistency, we use here our own classification. Note in any case that the difference between both (O9.7II in Hanson 2003, B0II according to our own classification) is minimal.
}

Tables 1 and 2 are the averages of the classifications attributed by each author.

The spectral types obtained for the new members of the region are encompassed within a very narrow range going from $\mathrm{O} 7$ to B2, with the vast majority of the members having subtypes between 09 and B1. In contrast, stars can be found in all the luminosity classes from I to V. Our sample includes both objects with featureless spectra in the near-infrared (Table 1) and objects observed to display lines of the Brackett series in emission (Table 2). Since we restricted our visible spectroscopy to targets with relatively bright magnitudes in the blue, we only expect classical Be stars in the latter category to be present in the sample observed in the visible. Indeed, none of the stars that we observed in the visible displays the strong infrared excess characteristic of Herbig Ae/Be stars (Hernández et al. 2005). On the other hand, of all the classical Be star candidates only one, star $\# 128$, also displays noticeable emission in the visible in the form of a faint emission core at the center of the Balmer lines.

\subsection{Spatial distribution}

Schematic plots with the spatial distributions of stars that we consider as likely members and non-members of the Cygnus OB2 halo are presented in Fig. 9. Candidate members are those listed in Tables 1-3, 9, as well as the young stellar object \#221. Both populations have clearly different distributions: the non-members are roughly uniformly spread out, as we may expect from a population composed by old stars. Such uniform distribution implies that there are no significant large-scale differences in extinction along the line of sight at least out to the typical distances of those stars, which as noted above are greater on the average than those of early-type stars of the same apparent magnitude.

The stars that we have considered as likely members, on the other hand, do display structure in their spatial distribution. Although OB stars are found all over the field, two areas of surface density greater than the average appear toward the (galactic) northwest (upper right in the left panel of Fig. 9) and toward the east (left in that same figure).

Figure 10 identifies the main structures known in the region, superimposed on a $1420 \mathrm{MHz}$ radio continuum map from the Canadian Galactic Plane Survey (Taylor et al. 2003) tracing the emission by ionized gas. A comparison to previously-known structures in that region shows that the northeastern concentration corresponds to the eastern boundary of the Cygnus OB9 association (e.g., Garmany \& Stencel 1992), and in particular to the cluster NGC 6910 (the tight concentration of stars near $l=$ $78.7, b=+2.0$, which are recovered by our candidate member selection procedure). On the eastern side, the broad enhancement of stellar surface density contains the chain of compact HII regions DR 17, DR 21 in the W75 complex, DR 23, and DR 22, all of which are embedded in the Cygnus X North complex. The reality of Cygnus $\mathrm{X}$ as a single physical entity, rather than as a chance alignment of unrelated structures lying at different distances along a spiral arm seen nearly end-on, has been widely debated in the literature (see, e.g., Odenwald \& Schwartz 1993). Recent detailed observations of the molecular gas in the region, mainly by Schneider et al. (2006, 2007), strongly argue for a real interconnection among these structures and present evidence for their interaction with the massive stars of Cygnus OB2. Based on those results, we consider here that DR 17, W75/DR 21, DR 22, and DR 23 are all structures belonging to Cygnus X and lying at the same distance as Cygnus OB2, although we note that a distance of 2-3 kpc to W75/DR21, significantly larger than the one 

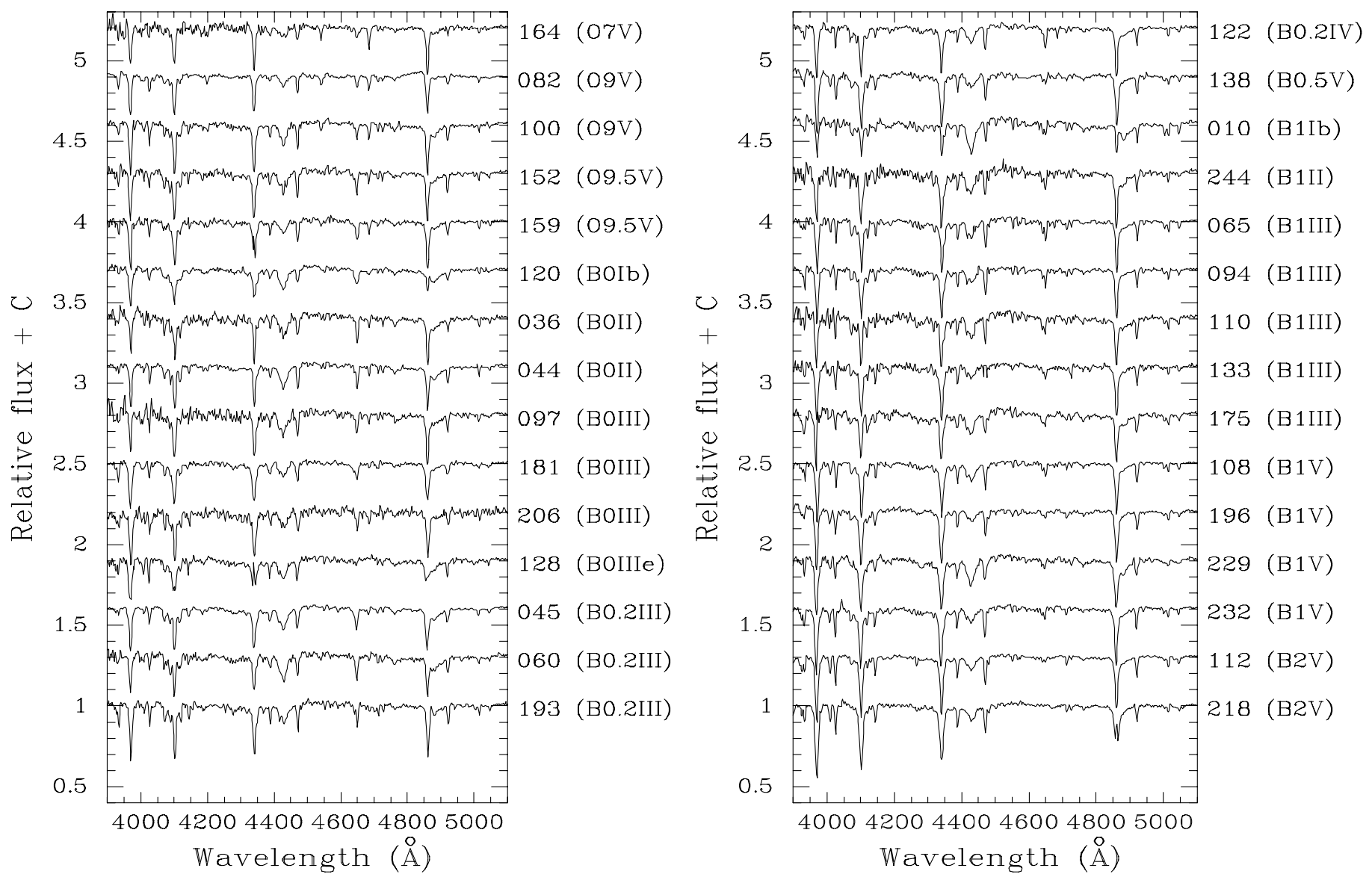

Fig. 8. Visible spectra of the stars for which a new MK spectral classification is given in this work. Note the strong interstellar feature centered at $4428 \AA$, due to the strong extinction. The main criteria used for classification are outlined in Sect. 4.3.
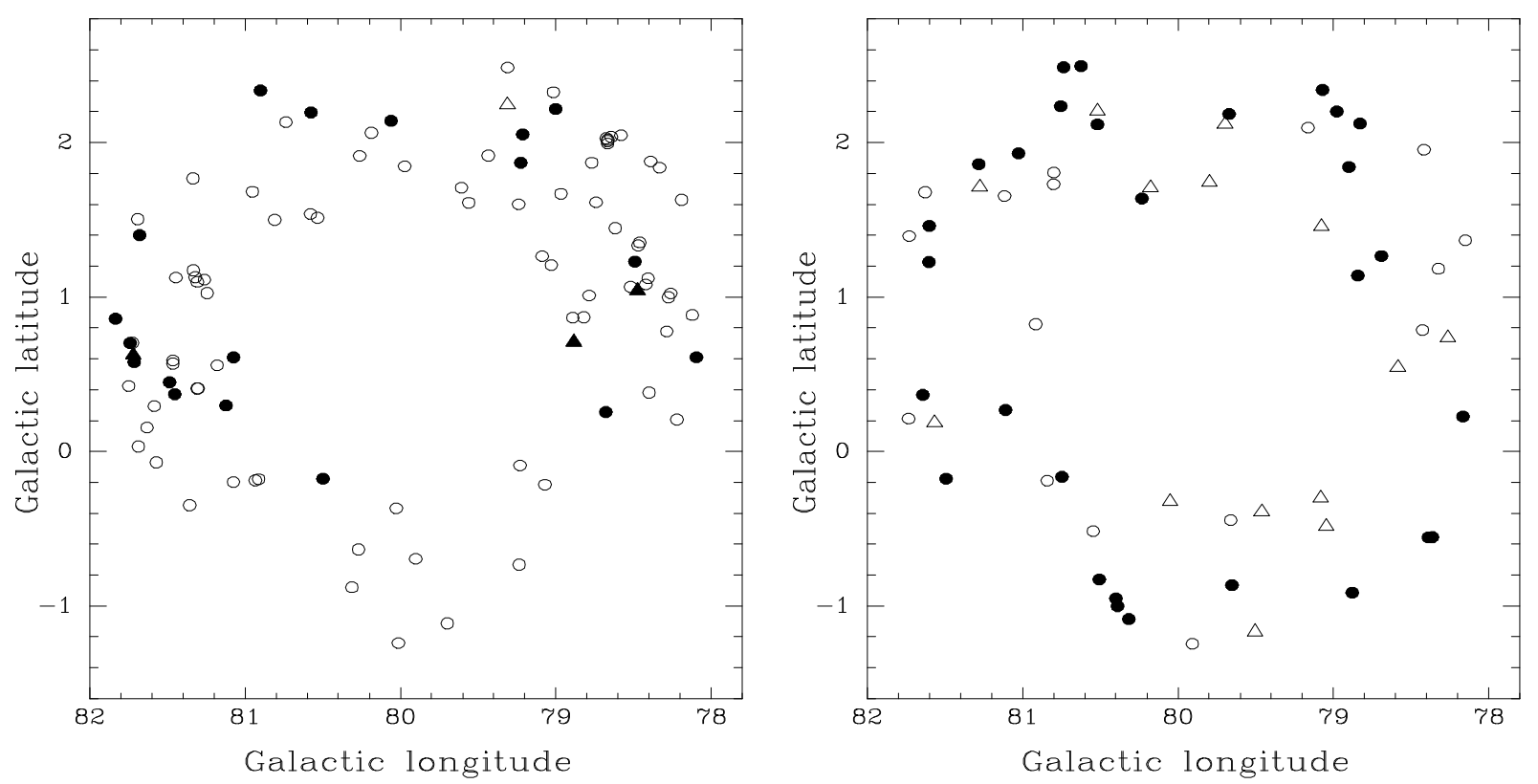

Fig. 9. Left panel: location of all the candidate members identified through their infrared spectra (Tables 1-3), including the protostellar candidates listed in Table 9 and star \#221. Symbols are as follows: open circles, stars with featureless near-infrared spectra; filled circles, stars with Brackett emission lines; filled triangles, massive young stellar objects; and open triangle, Wolf-Rayet star WR 142a. Right panel: same for three classes of non-members; open circles, red giant stars; filled circles, carbon stars; and open triangles, long-period variables. 


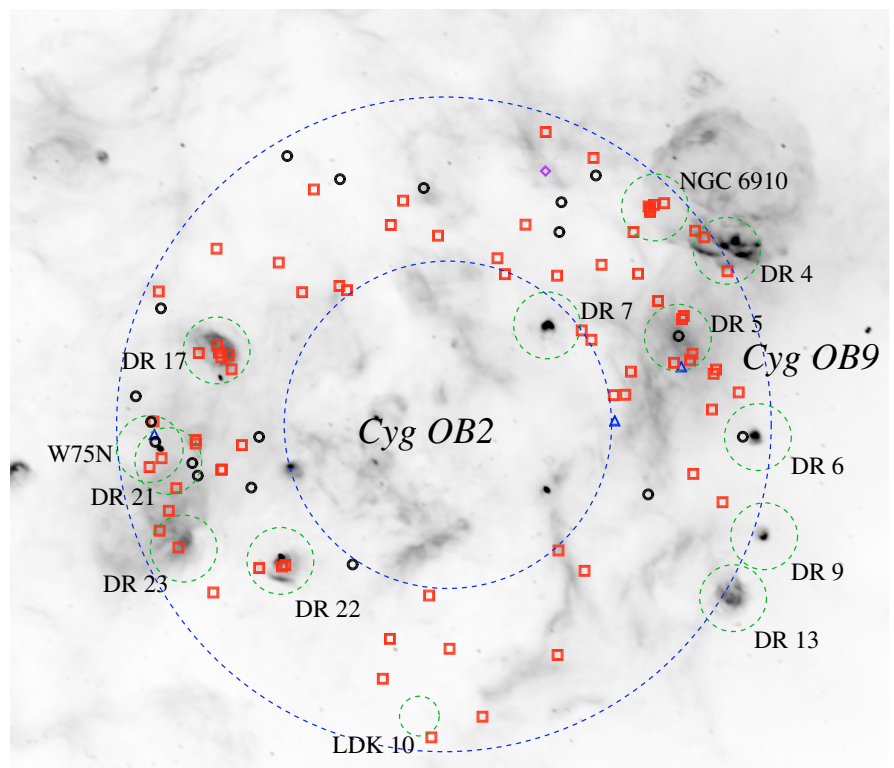

Fig. 10. Location of all the 98 candidate early-type stars overlaid on a radiocontinuum image of the region at $1420 \mathrm{MHz}$, from the Canadian Galactic Plane Survey (Taylor et al. 2003). Stars are represented by the following symbols: red squares, stars with featureless near-infrared spectra; black circles, stars with Brackett emission lines; blue triangles, massive young stellar objects; and diamond, Wolf-Rayet star WR 142a. The large inner and outer blue dashed circles are respectively $1^{\circ}$ and $2^{\circ}$ in radius, and they delimit the region in the outskirts of Cygnus OB2 covered by the present study. The location of the main HII regions, as well as the W75N star forming region and the NGC 6910 open cluster, are indicated. The HII regions noted here with prefix "DR" were identified by Downes \& Rinehart (1966). The prefix "LDK" refers to the list of embedded clusters of Le Duigou \& Knödlseder (2002). The position of the compact HII region DR 7 is marked here, although it is probably more distant and associated to the background Perseus arm (Comerón \& Torra 2001).

adopted here, has been often adopted in the literature (Kumar et al. 2007).

The concentration of early-type stars on the eastern side is particularly obvious at the position of DR 17, composed by the earliest-type members of the embedded clusters hosted by this HII region (Le Duigou \& Knödlseder 2002). However, it is more widespread and extends beyond the boundaries of the compact HII regions. A similar result has been reported recently by Kumar et al. (2007), who find that many young stars revealed by their infrared excesses in the Spitzer bands are located outside the boundaries of the main molecular complexes in the region. We note, in particular, the concentration of emission-line stars toward this general region, with a preference toward the surroundings of DR 21, although DR 21 itself is too obscured for its embedded stars to be included in our magnitude-limited sample (Nadeau et al. 1991). Almost all these stars have clear infrared excess placing them among the Herbig Ae/Be stars as discussed in Sect. 4.2, with the only exception of star \#118, appearing in the direction of $\mathrm{W} 75 \mathrm{~N}$ and whose near-infrared colors place it in the classical Be star locus. Only another candidate classical Be star is possibly associated with a compact HII region, namely star \#122 near DR 6, although the spatial coincidence between them is not precise.

The southwestern (lower right) part of the area covered by this study South of the galactic equator appears particularly devoid of early-type stars. This is interesting since that region coincides with Cygnus X South (Schneider et al. 2006), and recent stellar surface density estimates based on extinctioncorrected starcounts (Bontemps et al., in prep; preliminary results shown in Reipurth \& Schneider 2008) indicate an enhanced stellar density in that direction, which appears to be an extension of Cygnus OB2. The starcount enhancement detected by Bontemps et al. is contained within $1^{\circ}$ from the center of Cygnus OB2 and is in rough agreement with the distribution of earlytype stars in the same region found by Comerón et al. (2002, see Fig. 14 in that paper), which preferentially extends toward the west. The same distribution is well matched by that of A0V-A5V stars recently identified by Drew et al. (2008). As discussed by those authors, the magnitudes of these stars suggest an average age somewhat older than that of the Cygnus OB2 cluster if they lie at the distance of $1.45 \mathrm{kpc}$ assumed here, in agreement with the results of Hanson (2003), and it is most likely the lower mass counterpart of the sample discussed by Comerón et al. (2002). The fact that we detect no traces of it in our color-selected sample beyond the $1^{\circ}$ circle suggests that it either does not reach beyond that distance, or that its possible extension does not contain massive stars picked up by our selection criterion. On the other hand, ${ }^{13} \mathrm{CO}$ maps of that region (Simon et al., in prep.; preliminary results shown in Reipurth \& Schneider 2008) indicate the presence of large amounts of molecular gas in the zone where we do not detect a corresponding overdensity of earlytype stars. It thus appears that any star formation currently going on in that region of Cygnus $\mathrm{X}$ is not producing massive stars. It should be noted that this does not apply to the entire Cygnus $\mathrm{X}$ South region, which extends well beyond the boundaries of the area considered here and does contain massive embedded clusters and HII regions.

\subsection{Evolutionary status}

It is thus possible to identify three distinct components in the area targeted by this study. The first one is the population associated with the eastern edge of Cygnus OB9, particularly the cluster NGC 6910. The second component extends over the region occupied by the DR 17, W75/DR 21, DR 22, and DR 23 complexes in Cygnus X North. The third component is a distributed population whose members are stars that do not belong to either of those two groups, which may be related to the extended population in the central $1^{\circ}$-radius circle around Cygnus OB2 reported by Comerón et al. (2002) and Hanson (2003), and probably also by Drew et al. (2008).

The new availability of detailed spectral classifications for many stars in these three groups allows us to investigate in more detail their nature and evolutionary status. To this purpose we have adopted for each O-type star an effective temperature $T_{\text {eff }}$ based on the $T_{\text {eff }}$-spectral type calibration of Martins et al. (2005), using their observational effective temperature scale. To our knowledge no similar work is available to date covering the early B spectral interval, in particular the whole range of luminosity classes covered by our spectra. We have thus used

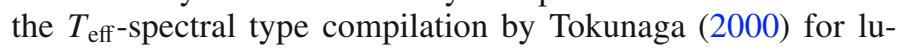
minosity classes I and V, applying a scaling factor to the temperatures so as to force agreement with Martins et al.'s (2005) observational temperature scale at spectral types $\mathrm{O} 9 \mathrm{I}$ and $09.5 \mathrm{~V}$ respectively. The calibrations of Tokunaga (2000) and Martins et al. (2005) closely match each other in the overlapping late-O range, implying that our arbitrary scaling factors deviate from unity by less than $4 \%$. Because the compilation of Tokunaga (2000) does not include luminosity class III earlytype stars, we have estimated their temperatures by means of 

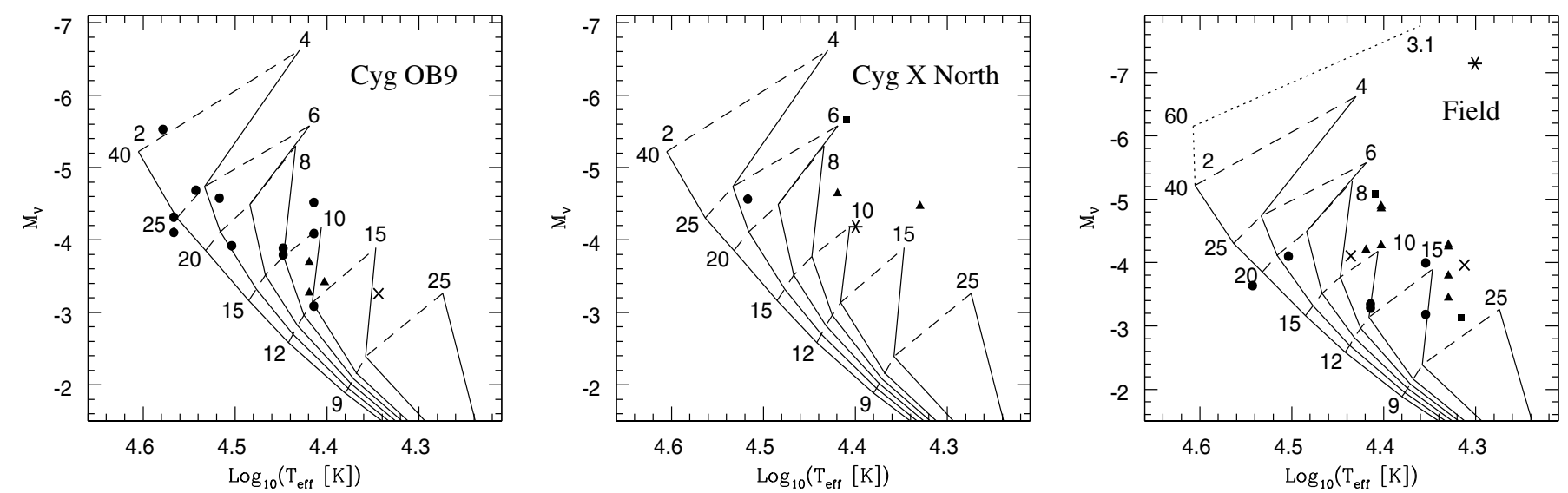

Fig. 11. Temperature-absolute magnitude diagram with the location of the stars for which spectral classification is available in each of the three regions discussed in Sect. 4.5. Circles indicate luminosity class V, crosses class IV, triangles class III, squares class II, and asterisks class I. The evolutionary tracks and isochrones from Lejeune \& Schaerer (2001) are plotted for comparison, highlighting the older age of the field population. We believe that most of the stars in the Cygnus X North area actually belong to the part of the field population lying along that line of sight.

a weighted interpolation between the temperatures adopted for classes I and V. The weight factor is derived once again by forcing agreement between the temperatures derived in this way for spectral type O9III and the corresponding value given by Martins et al. (2005). Approximate temperatures of B stars of luminosity classes II and IV have then been computed by averaging the temperatures adopted for stars of luminosity class I and III, and III and V, respectively. We estimate that the systematic errors introduced in this way are below $2000 \mathrm{~K}$ for all the spectral types represented in our sample. A summary of $T_{\text {eff }}$-spectral type calibrations for B-type stars presented by Fitzpatrick \& Massa (2005) shows that existing systematic uncertainties in this domain exceed this value. We thus consider that the ad-hoc procedure that we have described to assign effective temperatures to our stars does not introduce large additional uncertainties.

The large scatter in absolute magnitudes at a given spectral type among O and early-B stars (e.g., Jaschek \& Gómez 1998) cautions against the use of the spectral classification alone to derive the positions of our stars in the temperature-absolute magnitude diagram, and compare them to the predictions of evolutionary tracks to assess their evolutionary status. Instead, we have preferred to adhere to the underlying hypothesis already noted in Sect. 4.4 that all our stars have essentially the same distance modulus of $10.80 \mathrm{mag}$, and derive individual absolute magnitudes as

$M_{V}=K-10.80-0.659\left[(J-K)-(J-K)_{0}\right]+(V-K)_{0}$

where $(J-K)_{0}$ and $(V-K)_{0}$ are the intrinsic, unreddened color indices and the factor 0.659 is derived from the extinction law of Rieke \& Lebofsky (1985). We have used the intrinsic colors derived by Martins \& Plez (2006) for O stars and those compiled by Tokunaga (2000) for B stars. We estimate that interpolations needed to cover all the spectral types and luminosity classes in our sample introduce a systematic error in $M_{V}$ not exceeding $0.1 \mathrm{mag}$. The scatter in intrinsic colors among actual stars is likely to be a more important source of errors, as illustrated by the results of Robert et al. (2003).

The $T_{\text {eff }}-M_{V}$ diagrams are shown in Fig. 11 for the eastern edge of Cygnus OB9, the region in Cygnus X North around DR 21, and the field population. In these figures, we also plot the isochrones calculated by Lejeune \& Schaerer (2001) in their Case $e$ with solar metallicity, high mass loss and no rotation, for initial stellar masses $(60), 40,25,20,15,12$ and $9 M_{\odot}$ (solid lines) and stellar ages 2, (3.1), 4, 6, 8, 10, 15 and 25 Myr (dotted lines). Individual $T_{\text {eff }}$ and $M_{V}$ derived for each star in the different regions are listed in Table 10.

There are significant differences between the distributions of stars in the temperature-luminosity diagrams among the three components considered. The Cygnus OB9 region contains abundant O-type main sequence stars, particularly in the cluster NGC 6910 whose members display other signatures of youth (Delgado \& Alfaro 2000), and only four stars outside the main sequence (the B giants \#181, 193, and 206 and the B1.5IV star \#142) are found within this region in our sample. The class V O-type stars appear mostly above the main sequence, which may indicate that they lie at a shorter distance than that adopted by us. Garmany et al. (1992) find a very uncertain distance modulus $D M=10.0$ to NGC 6910 , in contrast with the better determined value for the rest of the association, $D M=11.0$. However, this latter value is in closer agreement with the distance found by Delgado \& Alfaro (2000) for NGC 6910 itself, $D M=11.2 \pm 0.2$, which solves the discrepancy noted by Garmany \& Stencel (1992) and is only marginally larger than that adopted by us. A value significantly shorter than $D M=10.8$ thus does not seem supported by other observations. The position of the class V O-type stars above the lowest isochrone may be at least partly due to binarity, as well as to rotation, whose effects can brighten $\mathrm{O}$ stars by as much as $\Delta M_{V}=0.6$ (Meynet $\&$ Maeder 2000) for an initial velocity of $200 \mathrm{~km} \mathrm{~s}^{-1}$.

The situation is less clear in Cygnus X North, as the higher extinction in the area prevented us from obtaining visible spectra of many stars. The presence of several Brackett emission-line stars with the infrared characteristics of Herbig Ae/Be stars suggests recent star formation over a widespread area. The five stars with spectral classification in the zone are all lightly reddened and may be on the near side of the complex. The luminosity classes (Ib-III) of four of them indicates that they are not related to the ongoing star formation in the area, with the possible exception of the O9V star \#100 that is very close to DR 21. It can be seen in Fig. 11 that a physical association of this star with DR 21 would support the distance adopted here, rather than the 2-3 kpc often used in the literature (Davis et al. 2007), as a greater distance would move it even further away from the main sequence. 
Table 10. Adopted temperatures and absolute magnitudes.

\begin{tabular}{|c|c|c|c|}
\hline Star & Sp. type & $M_{V}$ & $\log T_{\text {eff }}(\mathrm{K})$ \\
\hline Cygnus OB9 area 27 & $06.5 \mathrm{~V}$ & -5.53 & 4.578 \\
\hline 62 & O8V & -4.69 & 4.542 \\
\hline 68 & B1V & -4.52 & 4.414 \\
\hline 82 & $\mathrm{O} 9 \mathrm{~V}$ & -4.58 & 4.517 \\
\hline 101 & $\mathrm{O} 7 \mathrm{~V}$ & -4.32 & 4.567 \\
\hline 108 & B1V & -4.09 & 4.414 \\
\hline 137 & B $0.5 \mathrm{~V}$ & -3.80 & 4.448 \\
\hline 138 & B $0.5 \mathrm{~V}$ & -3.89 & 4.448 \\
\hline 142 & B1.5IV & -3.26 & 4.344 \\
\hline 159 & $09.5 \mathrm{~V}$ & -3.92 & 4.504 \\
\hline 164 & O7V & -4.10 & 4.567 \\
\hline 181 & B0III & -3.69 & 4.419 \\
\hline 193 & B0.2III & -3.42 & 4.403 \\
\hline 206 & B0III & -3.28 & 4.419 \\
\hline 232 & B1V & -3.09 & 4.414 \\
\hline Cygnus X North (DR 21) area 36 & B0II & -5.67 & 4.409 \\
\hline 65 & B1III & -4.47 & 4.329 \\
\hline 97 & B0III & -4.65 & 4.419 \\
\hline 100 & O9V & -4.57 & 4.517 \\
\hline 120 & B0Ib & -4.19 & 4.399 \\
\hline Field area 10 & B1Ib & -7.14 & 4.301 \\
\hline 44 & B0II & -5.08 & 4.409 \\
\hline 45 & B0.2III & -4.85 & 4.403 \\
\hline 50 & B3II & -4.03 & 4.191 \\
\hline 60 & B0.2III & -4.89 & 4.403 \\
\hline 64 & B0.2III & -4.27 & 4.403 \\
\hline 71 & B2IV & -3.96 & 4.312 \\
\hline 94 & B1III & -4.28 & 4.329 \\
\hline 110 & B1III & -4.25 & 4.329 \\
\hline 112 & B2V & -4.00 & 4.354 \\
\hline 122 & B0.2IV & -4.11 & 4.436 \\
\hline 128 & B0IIIe & -4.21 & 4.419 \\
\hline 133 & B1III & -3.80 & 4.329 \\
\hline 150 & O8V & -3.64 & 4.543 \\
\hline 152 & $09.5 \mathrm{~V}$ & -4.10 & 4.504 \\
\hline 175 & B1III & -3.45 & 4.329 \\
\hline 196 & B1V & -3.28 & 4.414 \\
\hline 218 & $\mathrm{~B} 2 \mathrm{~V}$ & -3.18 & 4.354 \\
\hline 229 & B1V & -3.35 & 4.414 \\
\hline 244 & B1II & -3.13 & 4.315 \\
\hline
\end{tabular}

In contrast, most of the population outside those two areas, which we consider as the field component, is lightly reddened, and visible spectroscopy is available for 20 of its members, of which 14 are found to have luminosity classes placing them above the main sequence. Of the remaining six stars, four are classified as B1V or B2V and thus main sequence lifetimes longer than $10 \mathrm{Myr}$; and only three stars, \#150 (O8V; Morgan et al. 1955), \#152 (O9.5V; this work) and WR 142a (WC8, for which Pasquali et al. (2002) estimate an age of at least 3 Myr) indicate more recent massive star formation. The first two are also the ones closest to the main sequence plotted in Fig. 11, in agreement with the common distance adopted for all our stars. Besides WR 142a, 7 out of 9 emission line stars in the region appear to be classical Be stars, with the only exceptions of stars \#178 and \#180. A comparison of the position of the stars belonging to the field component with the evolutionary tracks in the temperature-luminosity diagram indicates ages older than $6 \mathrm{Myr}$ for this population.

It may be noted that several stars classified as B1V or B2V appear up to 2 mag above the main sequence in the temperaturemagnitude diagrams for both Cygnus OB9 and the field population. This is considerably more than what one may expect from the effects of rotation, binarity, or the spread in intrinsic magnitudes within their spectral types. In fact such stars are not expected to be present in our sample, as normal main sequence stars later than B1V at the distance of Cygnus OB2 should have $K_{\mathrm{S}}$ magnitudes fainter than our target selection limit (see Sect. 2). In principle, these may be unrelated foreground stars at much closer distances. However, an inspection of their spectra in Fig. 8 shows interstellar features of a depth similar to those of the other stars in the region, indicating a similar level of extinction that is confirmed by their infrared photometry. A misclassification of the luminosity class thus appears as a more likely cause, which may be confirmed with better quality spectra. However, we have decided not to modify the spectral types given in this paper so as not to bias our classification with a posteriori knowledge.

\section{Discussion}

Our observations sample an interesting region where the outskirts of the Cygnus OB2 association merge with neighbor structures, allowing us to explore the possible existence of links among them and the history of star formation in the whole region. Such questions are rendered all the more relevant given the increasing evidence for their common distance (Schneider et al. 2007) and the possibility that the distinct structures recognized thus far are parts of a single association, particularly Cygnus 1, 8, and 9 (Mel'nik \& Efremov 1995).

One of our main results is the identification of an extended distribution of OB stars around Cygnus OB2 and probably beyond, selected through their near-infrared colors and confirmed on the basis of their near-infrared spectrum. Visible spectroscopy confirms the very high reliability of infrared spectroscopy at modest resolution and signal-to-noise ratio in identifying earlytype stars, as already found by Hanson (2003) when investigating the sample of candidates identified by Comerón et al. (2002). Our results also show a remarkable coincidence with those of Hanson (2003) in that the newly-identified early-type stars do not have, as a rule, spectral types as early as those at the center of Cygnus OB2. Instead, they are dominated by massive stars that have already evolved off the main sequence (i.e., giants, supergiants, and a Wolf-Rayet) or are thought to be at the end of their main sequence lifetime (the classical Be stars). Such evolved stars are dominant among the field component described in Sect. 4.5. Their presence also among the populations that we identified as belonging to the complex that includes DR 17, DR 21, DR 22, and DR 23, on one side, and Cygnus OB9, on the other, is not surprising since our allocation of stars to those groups is purely based on position. A certain level of contamination of those two components by stars actually belonging to the field population is thus to be expected.

What is the relationship between the field component and Cygnus OB2, if there is one? Hanson (2003) already pointed out that the spatial distribution of the evolved component differed markedly from that of the most massive stars of Cygnus OB2 in being much more evenly distributed over the studied area. In fact, based on both the wider spatial distribution and the gap in age Hanson suggested that the evolved component is unrelated to Cygnus OB2. Our new observations allow us to reevaluate this conclusion by extending the analysis of the spatial distribution to a larger area.

Unfortunately, the somewhat different selection of candidate early-B stars carried out in Comerón et al. (2002) and the present paper prevents a direct comparison between the stellar densities inside and outside the $1^{\circ}$ radius. It is however possible to perform the color selection of candidates over a much larger area 


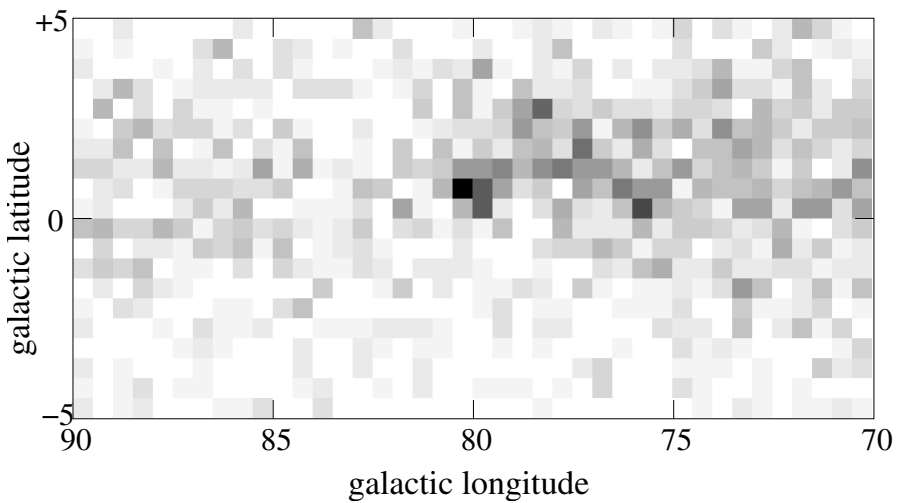

Fig. 12. Surface density of $O B$ stars selected according to the criteria $K<8.8,-0.15<(J-H)-1.70\left(H-K_{\mathrm{S}}\right)<0.20$. The shade of grey scales with the number of stars per square degree, ranging from zero (white) to 100 or more (black). The Cygnus OB2 central cluster is the black square near the center, with a density of 176 stars per square degree. A pedestal surface density of 12.5 stars per square degree has been subtracted from each cell to account for the estimated contamination by cool and foreground stars that are also selected by the color-based criteria.

that includes the central $1^{\circ}$ radius around Cygnus OB2, the region covered by this study, and the surrounding regions, using the results of Sect. 4.1 as a way to remove statistically the contamination by cool background stars and by foreground stars.

We have carried out this exercise over the area limited by $70^{\circ}<l<90^{\circ},-5^{\circ}<b<+5^{\circ}$, which has Cygnus OB2 near its center and includes the entire Cygnus OB9 region as well as parts of Cygnus OB1 to the West and Cygnus OB7 to the East. Stars were selected according to the criteria $K_{\mathrm{S}}<8.8$, $-0.15<(J-H)-1.70\left(H-K_{\mathrm{S}}\right)<0.20$. The results of the spectroscopic follow-up discussed in Sect. 4.1 indicate that these criteria select approximately $12.5 \mathrm{cool}$ (red giants, longperiod variables, and carbon stars) and foreground (mostly late B to F-type) stars per square degree, in addition to OB stars. We assume that these contaminants are uniformly distributed across the field, and subtract this number density from the total number density of selected objects. The result is displayed in Fig. 12, which is similar to the surface density maps presented by Knödlseder (2000) and Bontemps et al. (in prep., presented by Reipurth \& Schneider 2008). The main difference is that Fig. 12 is restricted to early-type stars; the surface densities are thus much lower and the resolution much coarser than in their contour maps. As one may expect, Cygnus OB2 is the most prominent feature appearing as a very tight concentration near the center of the figure, with a peak surface density reaching $176 \mathrm{OB}$ stars per square degree in the central 0.25 square degrees. The stellar density remains high toward the western half occupied by Cygnus OB9 next to Cygnus OB2, and then Cygnus OB1. The easternmost quarter of the figure is occupied by the more nearby association Cygnus OB7 (Garmany \& Stencel 1992). Despite the overall similarity, there are significant differences between our map and the general starcount-based map of Bontemps et al.: Cygnus OB9 appears much more prominently in our map, whereas the DR 17/DR 21/DR 22/DR23 region, while easily recognizable as a stellar density enhancement in our Fig. 10, stands out much more clearly in Bontemps et al.'s map.

Interestingly, Fig. 12 shows that a non-zero surface density of early-type star candidates pervades the region even outside the boundaries of the already-known OB associations. By extrapolating the results obtained in the area between $1^{\circ}$ and $2^{\circ}$ from the center of Cygnus OB2, we tentatively identify this as the field population mainly composed of evolved stars, noting that this population does not appear to be associated with Cygnus OB2 nor with any other specific feature in the region. The massive precursors required by this widespread field population suggests that massive star formation has been taking place in the Cygnus region for a time exceeding $10 \mathrm{Myr}$, and long before the formation of the currently observed associations like Cygnus OB2, whose age is less than 4 Myr (Massey et al. 1995). The fact that such a population appears as an extended component with no apparent concentration is not entirely surprising, given the high density of $\mathrm{OB}$ associations in the region, if we assume that this density was similarly high in the past. The typical center-tocenter distance among the four associations currently defined in the galactic longitude interval 74 $<l<82^{\circ}$ (Cygnus OB1, $\mathrm{OB} 2, \mathrm{OB} 8$, and $\mathrm{OB} 9$ ) is $\sim 2^{\circ} 5$. At the distance of $1.45 \mathrm{kpc}$ adopted throughout this paper, an internal velocity dispersion of $3.5 \mathrm{~km} \mathrm{~s}^{-1}$ would suffice to make them overlap after $10 \mathrm{Myr}$ if they are gravitationally unbound, even if they occupied a small initial volume. This velocity dispersion is comparable to the internal velocity dispersion derived for members of Cygnus OB2 by Kiminki et al. (2007), $2.44 \pm 0.07 \mathrm{~km} \mathrm{~s}^{-1}$. Moreover it can be regarded as an upper limit, since even very young OB associations like Cygnus OB9 occupy a sizeable volume comparable to the center-to-center angular distance quoted above, and the field population contains members older than 10 Myr leaving more time for dispersal of the original associations. This population may be the equivalent to an extended component also observed in other galaxies (Pasquali \& Castangia 2008), and it can thus be accounted for by assuming a previous generation of OB associations, now dispersed, with characteristic similar to those of the current associations. It appears unlikely that the field population has its origin in bound clusters having become unbound in their early evolutionary stages (Lada \& Lada 2003): recent observational evidence discussed by Gieles \& Bastian (2008) indicates dispersal timescales at least one order of magnitude longer than the age of the field population in a wide range of environments, including our galactic neighborhood. Our results rather suggest that those massive $\mathrm{OB}$ stars originate in either isolation or unbound aggregates.

The sustained massive star forming activity in Cygnus must have been essential in arranging the large-scale distribution of the interstellar gas and in driving the current generation of star formation. It may also be at the origin of the large Cygnus Supperbubble observed in X rays (Cash et al. 1980; Bochkarev \& Sitnik 1985; Uyaniker et al. 2001) and possibly related phenomena such as the apparent large-scale expansion pattern noted by Comerón et al. (1998) in the proper motions of stars in the area.

\section{Conclusions}

We have extended previous work carried out mainly by Comerón et al. (2002) and Hanson (2003) in characterizing the population of early-type stars in the vicinity of Cygnus OB2. We have done this by identifying a magnitude-limited sample of candidate early-type stars through near-infrared imaging in the area between $1^{\circ}$ and $2^{\circ}$ from the center of Cygnus OB2, confirming them through near-infrared spectroscopy, and providing an accurate spectral classification of many of them through spectroscopy in the visible. Our main results can be summarized as follows:

- The studied area is rich in early-type stars, including evolved massive emission-line stars, likely Herbig Ae/Be stars, and 
some deeply-embedded massive young stellar objects. As in previous studies, a color-based selection criterion is found to be efficient in selecting candidate early-type stars, although with a considerable degree of contamination by other types of objects, mainly cool stars and foreground late-B, A, and F stars. Near-infrared spectroscopy at modest resolution and signal-to-noise ratio suffices to identify the actual emissionline stars.

- Our early-type selection criteria recovers many stars belonging to the Cygnus OB9 association and its cluster NGC 6910, which are partly included in the studied area. We also find a significant number of reddened early-type stars and Herbig Ae/Be star candidates in the area occupied by the Cygnus X North complex, which includes the compact HII regions DR 17, DR 21, DR 22, and DR 23. The massive star distribution is, however, not confined to the HII regions.

- Many early-type stars are found not to be associated with any known structure. Visible spectroscopy is available for most of this field population, and shows that it is strongly dominated by giant and supergiant early-B stars. The characteristics of this population appear to be the same as discussed by Hanson (2003) for the additional early-type stars identified by Comerón et al. (2002) in the vicinity of Cygnus OB2. It most probably represents a population significantly older than the massive stellar cluster at the center of Cygnus OB2.

- We have extended the color-based selection criterion to estimate the surface density of early-type candidates in an area of 200 square degrees centered on Cygnus OB2, statistically correcting for the unrelated background and foreground populations. We find that the field population is not particularly related to Cygnus OB2 nor to any other structure of the region, being all-pervasive. The need for massive precursors to produce this extended field population suggests that massive star formation in the whole Cygnus region has proceeded for a long time, well before the currently identified OB associations were formed. Such interpretation rests on the assumption underlying this work that all the early-type stars are located at similar distances, and it would be challenged if the field population were either foreground or background to the OB associations, or if it spread over a wide range of distances. Although this possibility needs to be clarified by future studies, we do not find evidence in our temperatureabsolute magnitude diagrams for a systematic difference between the distance to the members of this population and the adopted distance of Cygnus OB2.

We believe that the present study conclusively dismisses the case for a large extent of Cygnus OB2 much beyond the boundaries of its central concentration, which was already suggested by Garmany \& Stencel (1992) and supported by Comerón et al. (2002), but then questioned by Hanson (2003). Previous studies such as those by Massey \& Thompson (1991) and Knödlseder (2000) clearly show that most of the massive stellar content of the association still awaits identification and classification (see also Kiminki et al. 2007, for recent work on the confirmation of new $\mathrm{OB}$ members in the inner regions of Cygnus OB2). Our results indicate that future studies of Cygnus OB2 aiming to characterize its upper main sequence should concentrate on heavily-reddened members near its core, rather than on identifying new members at large distances from it.

We note that over half of the early-type stars for which we have obtained confirming near-infrared spectroscopy do not yet have a detailed spectral classification available. This is particularly important for the new candidates identified in Cygnus X North, whose proximity to compact HII regions, some of which contain embedded clusters, leads us to suspect a young age for most of them. If confirmed, the extent and composition of this group may warrant its status as an $\mathrm{OB}$ association in its own right, although we feel that a better characterization is still needed before taking that step. On the other hand, the as yet unclassified stars located elsewhere offer a potential for the discovery of new interesting members deserving detailed study, such as WR $142 \mathrm{a}$ or $\mathrm{BD}+43^{\circ} 3654$. The fact that the vast majority of stars that form the basis for the present study are recognized here as early-type members of the region for the first time is a reminder of the large amount of observational work still needed to characterize the stellar population in the Cygnus region. The importance of this task is stressed by the rapid progress being made at different wavelengths in the characterization of its interstellar content.

Acknowledgements. Once again it is a pleasure to thank the staff of the Calar Alto observatory, and especially Santos Pedraz and Ana Guijarro, for their unfailingly competent and friendly support while observing on Calar Alto. We are very thankful to Bo Reipurth and Nicola Schneider for making available to us their chapter on the Cygnus region for the Handbook of Star Forming Regions prior to publication. We also thank the anonymous referee for constructive comments that helped improve both the style and content of this paper. F.C. warmly acknowledges the hospitality of the Vatican Observatory during the preparation of this paper. A.P. acknowledges support from the OPTICON Network. J.T. and F.F. acknowledge support by the Spanish Ministry of Science and Technology under contract AYA2006-15623-C02-02. This research has made use of the SIMBAD database operated at CDS, Strasbourg, France. It also makes use of data products from the Two Micron All Sky Survey, which is a joint project of the University of Massachusetts and the Infrared Processing and Analysis Center/California Institute of Technology, funded by the National Aeronautics and Space Administration and the National Science Foundation; and of data from the Canadian Galactic Plane Survey, a Canadian project with international partners, supported by the Natural Sciences and Engineering Research Council.

\section{References}

Adams, F. C., Lada, C. J., \& Shu, F. H. 1987, ApJ, 312, 788 Behr, B. B. 2003, ApJS, 149, 101

Benz, A. O., Stäuber, P., Bourke, T. L., et al. 2007, A\&A, 475, 549

Bica, E., Dutra, C. M., \& Barbuy, B. 2003, A\&A, 397, 177

Bidelman, W. P. 1957, PASP, 69, 147

Bochkarev, N. G., \& Sitnik, T. G. 1985, Ap\&SS, 108, 237

Carruthers, G. R., \& Page, T. 1984, ApJS, 54, 271

Cash, W., Charles, P., Bowyer, S., et al. 1980, ApJ, 238, L71

Chen, P.-S., \& Chen, W.-P. 2003, AJ, 125, 2215

Chlebowski, T., Harnden, F. R., \& Sciortino, S. 1989, ApJ, 341, 427

Comerón, F. \& Pasquali, A. 2005, A\&A, 430, 541

Comerón, F., \& Pasquali, A. 2007, A\&A, 467, L23

Comerón, F., \& Torra, J. 2001, A\&A, 375, 539

Comerón, F., Torra, J., \& Gómez, A. E. 1998, A\&A, 330, 975

Comerón, F., Pasquali, A., Rodighiero, G., et al. 2002, A\&A, 389, 874

Davis, C. J., Kumar, M. S. N., Sandell, G., et al. 2007, MNRAS, 374, 29

Delgado, A. J., \& Alfaro, E. J. 2000, AJ, 119, 1848

Demers, S., \& Battinelli, P. 2007, A\&A, 473, 143

Dolidze, M. V. 1975, Bull. Abastumian Astroph. Obs., 47, 3

Downes, D., \& Rinehart, R. 1966, ApJ, 144, 937

Drew, J. E., Greimel, R., Irwin, M. J., \& Sale, S. E. 2008, MNRAS, submitted

Drilling, J. S., \& Landolt, A. U. 2000, in Allen's Astrophysical Quantities, ed. A. N. Cox (AIP Press)

Dullemond, C. P., Dominik, C., \& Natta, A. 2001, ApJ, 560, 957

Ekström, S., Meynet, G., Maeder, A., \& Barblan, F. 2008, A\&A, 478, 467

Fabricius, C., \& Makarov, V. V. 2000, A\&A, 356, 14

Ferraro, F. R., Montegriffo, P., Origlia, L., \& Fusi Pecci, F. 2000, AJ, 119, 1282

Fitzpatrick, E. L., \& Massa, D. 2005, AJ, 129, 1642

Garmany, C. D., \& Stencel, R. E. 1992, A\&AS, 94, 211

Gieles, M., \& Bastian, N. 2008, A\&A, 482, 165

Giuricin, G., Mardirossian, F., \& Mezzetti, M. 1983, A\&AS, 54, 211

Griffith, M., Langston, G., Heflin, M., Conner, S., \& Burke, B. 1991, ApJS, 75, 801

Guetter, H. H. 1968, PASP, 80, 197 
Hanson, M. M. 2003, ApJ, 597, 957

Hanson, M. M., Conti, P. S., \& Rieke, M. J. 1996, ApJS, 107, 281

Hernández, J., Calvet, N., Hartmann, L., et al. 2005, AJ, 129, 856

Hoag, A. A., \& Applequist, N. L. 1965, ApJS, 12, 215

Herrero, A., Corral, L. J., Villamariz, M. R., \& Martín, E. L. 1999, A\&A, 348, 542

Jaschek, C., \& Gómez, A. E. 1998, A\&A, 330, 619

Kiminki, D. C., Kobulnicky, H. A., Kinemuchi, K., et al. 2007, ApJ, 664, 1102

Knapp, G. R., Pourbaix, D., Platais, I., \& Jorissen, A. 2003, A\&A, 403, 993

Knödlseder, J. 2000, A\&A, 360, 539

Knödlseder, J. 2003, in A Massive Star Odyssey: From Main Sequence to Supernova, ed. K. van der Hucht, A. Herrero, \& C. Esteban, ASP Conf. Ser., IAU Symp. 212

Kohoutek, L., \& Wehmeyer, R. 1999, A\&AS, 134, 255

Kumar, M. S. N., Davis, C. J., Grave, J. M. C., \& Ferreira, B. 2007, MNRAS, 374,54

Lada, C. J., \& Lada, E. A. 2003, ARA\&A, 41, 57

Lançon, A., \& Wood, P. R. 2000, A\&AS, 217, 271

Le Duigou, J.-M., \& Knödlseder, J. 2002, A\&A, 392, 869

Lejeune, T., \& Schaerer, D. 2001, A\&A, 366, 53

Martins, F., \& Plez, B. 2006, A\&A, 457, 637

Martins, F., Schaerer, D., \& Hillier, D. J. 2005, A\&A, 436, 1049

Massey, P., \& Thompson, A. B. 1991, AJ, 101, 1408

Massey, P., Johnson, K. E., \& Degioia-Eastwood, K. 1995, ApJ, 454, 151

McSwain, M. V., \& Gies, D. R. 2007, in Active OB-Stars: Laboratories for Stellar and Circumstellar Physics, ed. S. Štefl, S. P. Owocki, \& A. T. Okazaki, ASP Conf. Ser.

Melikian, N. D., \& Shevchenko, V. S. 1990, Astrofizika, 32, 169

Mel'nik, A. M., \& Efremov, Y. N. 1995, Astron. Lett. 21, 10

Merrill, P. W., \& Burwell, C. G. 1950, ApJ, 112, 72

Meynet, G., \& Maeder, A. 2000, A\&A, 361, 101

Monet, D. G., Levine, S. E., Canzian, B., Ables, H. D., \& Bird, A. R. 2003, AJ, 125,984

Morgan, W. W., Code, A. D., \& Whitford, A. E. 1955, ApJS, 2, 41

Morris, P. W., Eenens, P. R. J., Hanson, M. M., Conti, P. S., \& Blum, R. D. 1996, ApJ, 470, 597

Nadeau, D., Riopel, M., \& Geballe, T. R. 1991, ApJ, 372, L103
Nakashima, J.-I., \& Deguchi, S. 2003, PASJ, 55, 229

Nassau, J. J., \& Morgan, W. W. 1952, ApJ, 115, 475

Neckel, H. 1958, ApJ, 128, 510

Nordstrøm, B., Mayor, M., Andersen, J., et al. 2004, A\&A, 418, 989

Odenwald, S. F., \& Schwartz, P. R. 1993, ApJ, 405, 706

Oliva, E., \& Origlia, L. 1992, A\&A, 254, 466

Pasquali, A., \& Castangia, P. 2008, MNRAS, 385, 468

Pasquali, A., Comerón, F., Gredel, R., Torra, J., \& Figueras, F. 2002, A\&A, 396, 533

Poelman, D. R., \& van der Tak, F. F. S. 2007, A\&A, 475, 949

Reid, I. N., van Wyk., F., Marang, F., et al. 2001, MNRAS, 325, 931

Reipurth, B., \& Schneider, N. 2008, in Handbook of Star Formation Regions, ed. B. Reipurth (ASP), in press

Rieke, G. H., \& Lebofsky, M. J. 1985, ApJ, 288, 618

Robert, C., Pellerin, A., Aloisi, A., et al. 2003, ApJS, 144, 21

Schaerer, D., \& de Koter, A. 1997, A\&A, 322, 598

Schneider, N., Bontemps, S., Simon, R., et al. 2006, A\&A, 458, 855

Schneider, N., Simon, R., Bontemps, S., Comerón, F., \& Motte, F. 2007, A\&A, 474,873

Sigut, T. A. A., \& Jones, C. E. 2007, ApJ, 668, 481

Straizys, V., Meistas, E., Vansevicius, V., \& Goldberg, E. P. 1989 Vilnius Obs. Bull. 83, 3

Taylor, A. R., Goss, W. M., Coleman, P. H., van Leeuwen, J., \& Wallace, B. J. 1996, ApJS, 107, 239

Taylor, A. R., Gibson, S. J., Peracaula, M., et al. 2003, AJ, 125, 3145

Tokunaga, A. T. 2000, in Allen's Astrophysical Quantities, ed A. N. Cox (AIP Press)

Uyaniker, B., Fürst, E., Reich, W., Aschenbach, B., \& Wielebinski, R. 2001, A\&A, 371, 675

Vacca, W. D., Garmany, C. D., \& Shull, J. M. 1996, ApJ, 460, 914

Vijapurkar, J., \& Drilling, J. S. 1993, ApJS, 89, 293

Walborn, N. R. 1971, ApJS, 23, 257

Walborn, N. R., \& Fitzpatrick, E. L. 1990, PASP, 102, 1094

Walker, G. A. H., \& Hodge, S.M. 1968, PASP, 80, 290

Weinberg, M. D., \& Nikolaev, S. 2001, ApJ, 548, 712

Wendker, H. J., Higgs, L. A., \& Landecker, T. L. 1991, A\&A, 241, 551

Zorec, J., \& Briot, D. 1997, A\&A, 318, 443 
F. Comerón et al.: The outskirts of Cygnus OB2, Online Material p 1

Table 1. Stars with reddened, featureless spectra.

\begin{tabular}{|c|c|c|c|c|c|c|c|c|}
\hline Star & $\alpha(2000)$ & $\delta(2000)$ & $J$ & $H$ & $K_{\mathrm{S}}$ & Vis. classification & Other IDs & Notes \\
\hline 10 & 203142.15 & 422553.2 & 6.212 & 5.467 & 5.039 & $\mathrm{~B} 1 \mathrm{Ib}$ & & \\
\hline 33 & 202527.27 & 402400.1 & 7.200 & 6.852 & 6.652 & & BD+39 4179 & \\
\hline 36 & 203820.40 & 415656.4 & 7.839 & 7.086 & 6.686 & B0II & & \\
\hline 37 & 203915.58 & 421710.9 & 8.870 & 7.554 & 6.830 & & & 1 \\
\hline 39 & 203508.66 & 422557.0 & 9.359 & 7.801 & 6.966 & & & 2 \\
\hline 42 & 203542.96 & 422941.9 & 8.222 & 7.426 & 6.993 & & & 3 \\
\hline 44 & 203108.39 & 420242.3 & 7.815 & 7.298 & 7.029 & BOII & A41 & 4 \\
\hline 45 & 203202.20 & 421226.1 & 7.534 & 7.264 & 7.049 & B0.2III & BD+41 3794 & \\
\hline 60 & 202752.95 & 414406.8 & 8.152 & 7.580 & 7.271 & B0.2III & & \\
\hline 62 & 202322.84 & 400922.5 & 7.581 & 7.421 & 7.286 & & HD 229202 & 5 \\
\hline 65 & 203510.62 & 422022.6 & 7.829 & 7.510 & 7.326 & B1III & LS III +42 17 & 6 \\
\hline 70 & 203924.00 & 402939.3 & 8.734 & 7.862 & 7.373 & & & \\
\hline 72 & 202610.44 & 395151.9 & 8.745 & 7.847 & 7.388 & & & \\
\hline 74 & 203233.51 & 424725.5 & 8.963 & 7.977 & 7.443 & & & \\
\hline 75 & 204105.74 & 395519.5 & 8.526 & 7.806 & 7.448 & & & \\
\hline 82 & 202506.52 & 403549.6 & 7.931 & 7.650 & 7.490 & $\mathrm{O} 9 \mathrm{~V}$ & $\mathrm{BD}+404159$ & \\
\hline 93 & 204029.78 & 420313.7 & 9.243 & 8.156 & 7.619 & & & 7 \\
\hline 94 & 202533.19 & 404844.5 & 8.337 & 7.900 & 7.639 & B1III & & \\
\hline 96 & 202646.79 & 400557.0 & 8.955 & 8.073 & 7.655 & & & 8 \\
\hline 97 & 203821.72 & 415706.8 & 8.755 & 8.037 & 7.680 & BOIII & & \\
\hline 100 & 203945.06 & 420607.8 & 8.470 & 7.984 & 7.712 & O9V & & \\
\hline 101 & 202717.57 & 394432.6 & 8.100 & 7.822 & 7.727 & & LS II +3953 & 9 \\
\hline 108 & 202314.54 & 404519.3 & 8.157 & 7.932 & 7.783 & B1V & $\mathrm{BD}+404149$ & 10 \\
\hline 110 & 202302.91 & 413346.7 & 8.758 & 8.140 & 7.825 & B1III & & \\
\hline 129 & 202929.76 & 392122.4 & 9.538 & 8.523 & 8.003 & & & \\
\hline 130 & 204033.68 & 402245.1 & 9.775 & 8.606 & 8.028 & & & \\
\hline 131 & 202845.54 & 401704.0 & 9.239 & 8.456 & 8.043 & & & \\
\hline 132 & 203717.74 & 415631.4 & 9.092 & 8.379 & 8.053 & & & \\
\hline 133 & 202749.25 & 401700.4 & 8.705 & 8.310 & 8.079 & B1III & & \\
\hline 134 & 203944.16 & 421842.6 & 9.534 & 8.580 & 8.094 & & & 11 \\
\hline 135 & 203352.19 & 394726.5 & 9.271 & 8.482 & 8.105 & & & \\
\hline 137 & 202307.58 & 404609.0 & 8.480 & 8.244 & 8.126 & & & 10,12 \\
\hline 138 & 202405.15 & 404603.6 & 8.621 & 8.310 & 8.127 & B $0.5 \mathrm{~V}$ & LS III +40 15 & \\
\hline 140 & 203934.10 & 411656.5 & 10.080 & 8.800 & 8.140 & & & 13 \\
\hline 143 & 203522.96 & 422202.4 & 9.417 & 8.613 & 8.155 & & & 14 \\
\hline 146 & 202307.30 & 404655.2 & 8.504 & 8.254 & 8.206 & & & 10 \\
\hline 152 & 202737.87 & 411546.8 & 9.172 & 8.581 & 8.264 & $09.5 \mathrm{~V}$ & & \\
\hline 156 & 204115.71 & 415206.9 & 10.218 & 8.988 & 8.289 & & & 15 \\
\hline 159 & 202624.88 & 400141.4 & 9.044 & 8.569 & 8.322 & $09.5 \mathrm{~V}$ & & 16 \\
\hline 164 & 202619.75 & 395142.5 & 9.344 & 8.723 & 8.349 & $\mathrm{O} 7 \mathrm{~V}$ & & \\
\hline 169 & 203940.24 & 411734.0 & 10.329 & 9.098 & 8.389 & & & 17 \\
\hline 175 & 202902.47 & 423116.0 & 9.056 & 8.626 & 8.428 & B1III & & \\
\hline 181 & 202611.89 & 400222.4 & 9.282 & 8.746 & 8.464 & BOIII & & 18 \\
\hline 183 & 202918.68 & 393615.8 & 9.793 & 8.910 & 8.471 & & & 19 \\
\hline 185 & 202250.95 & 411350.7 & 9.785 & 8.973 & 8.519 & & & \\
\hline 186 & 203452.07 & 425508.1 & 9.615 & 8.901 & 8.524 & & & \\
\hline 189 & 203350.76 & 395938.7 & 9.675 & 8.925 & 8.541 & & & \\
\hline 190 & 204112.17 & 420123.7 & 10.726 & 9.332 & 8.548 & & & 20 \\
\hline 193 & 202254.49 & 402331.7 & 9.140 & 8.753 & 8.553 & B0.2III & & \\
\hline 196 & 202739.82 & 404038.6 & 8.938 & 8.673 & 8.579 & B1V & & \\
\hline 202 & 202831.87 & 401342.7 & 10.601 & 9.299 & 8.613 & & & \\
\hline 204 & 203529.68 & 421624.3 & 10.669 & 9.308 & 8.618 & & & 21 \\
\hline 205 & 203518.14 & 422348.4 & 10.052 & 9.075 & 8.620 & & & 22 \\
\hline 206 & 202528.89 & 401254.2 & 9.058 & 8.750 & 8.627 & B0III & LS III +40 32 & 23 \\
\hline 209 & 203934.16 & 394502.3 & 10.015 & 9.116 & 8.634 & & & \\
\hline 219 & 202254.30 & 402741.2 & 9.085 & 8.806 & 8.700 & & HD 229167 & \\
\hline 220 & 203632.51 & 393647.2 & 10.054 & 9.189 & 8.713 & & & 24 \\
\hline 222 & 203730.82 & 402752.0 & 10.580 & 9.351 & 8.723 & & & \\
\hline 227 & 203805.37 & 421114.2 & 9.964 & 9.162 & 8.740 & & & \\
\hline 229 & 202720.99 & 412126.2 & 9.460 & 8.981 & 8.746 & B1V & & \\
\hline
\end{tabular}


F. Comerón et al.: The outskirts of Cygnus OB2, Online Material p 2

Table 1. continued.

\begin{tabular}{ccccccccc}
\hline \hline Star & $\alpha(2000)$ & $\delta(2000)$ & $J$ & $H$ & $K_{\mathrm{S}}$ & Vis. classification & Other IDs & Notes \\
\hline 230 & 204143.65 & 413145.2 & 10.090 & 9.224 & 8.767 & & & \\
232 & 202258.94 & 404539.4 & 9.131 & 8.905 & 8.774 & B1V & & 26 \\
242 & 203826.96 & 422744.8 & 9.876 & 9.145 & 8.823 & & & \\
244 & 203106.24 & 415932.4 & 9.615 & 9.128 & 8.824 & B1II & & \\
246 & 202640.87 & 405946.9 & 11.198 & 9.652 & 8.829 & & & 27 \\
250 & 204010.22 & 412353.5 & 10.901 & 9.565 & 8.852 & & & \\
\hline
\end{tabular}

Notes

1: In DR 21, possibly associated to the radiosource WSRTGP 2037+4206 (Taylor et al. 1996).

2: In DR 17. 3: In DR 17. Brightest star in cluster 14 of Le Duigou \& Knödlseder (2002).

4: In Comerón et al. (2002); classified as O9.7II by Hanson (2003).

5: Classified as O8V: by Morgan et al. (1955); X-ray emission (1989).

6: Possibly related to the HII region G081.3+01.1 in DR17.

7: In DR 23. Brightest star in cluster 15 of Le Duigou \& Knödlseder (2002).

8: Possibly associated to DR 5.

9: Classified as O7V: by Vijapurkar \& Drilling (1993).

10: Member of cluster NGC 6910.

11: In DR 21.

12: Classified as B0.5V by Hoag \& Applequist (1965)

13: In DR 22, member of cluster 11 of Le Duigou \& Knödlseder (2002).

14: In DR 17, member of cluster 12 of Le Duigou \& Knödlseder (2002).

15: In cluster associated to DR 23 (Bica et al. 2003).

16: Possibly associated to DR 5.

17: In DR 22.

18: Possibly associated to DR 5.

19: Possibly associated to the radiosource MITGJ2029+3936 (Griffith et al. 1991).

20: Possibly associated to DR 23.

21: In DR 17.

22: In DR 17.

23: Possibly associated to DR 5.

24: Probably associated to IRAS 20346+3926A.

25: In cluster NGC 6910 (Delgado \& Alfaro 2000).

26: In DR 21.

27: Possibly associated to DR 22. 
Table 2. Emission line stars.

\begin{tabular}{|c|c|c|c|c|c|c|c|c|}
\hline Star & $\alpha(2000)$ & $\delta(2000)$ & $J$ & $\bar{H}$ & $K_{\mathrm{S}}$ & Vis. classification & Other IDs & Notes \\
\hline 46 & 202438.42 & 411409.9 & 8.574 & 7.762 & 7.055 & & AS 415 & 1 \\
\hline 48 & 202406.20 & 412533.9 & 9.220 & 8.077 & 7.089 & & WR142a & 2 \\
\hline 66 & 202652.54 & 415838.2 & 8.703 & 7.880 & 7.340 & & & 3 \\
\hline 67 & 203644.24 & 415317.4 & 9.077 & 7.986 & 7.348 & & & \\
\hline 112 & 202815.21 & 422539.1 & 8.286 & 7.999 & 7.838 & B2V & $\mathrm{BD}+413762$ & \\
\hline 116 & 203808.27 & 423836.5 & 12.058 & 9.687 & 7.874 & & & 4,5 \\
\hline 118 & 203516.63 & 425047.1 & 8.239 & 8.018 & 7.906 & & BD+42 3793 & 6 \\
\hline 120 & 203859.18 & 420239.4 & 8.686 & 8.191 & 7.909 & B0Ib & AS 434 & 7 \\
\hline 122 & 202725.52 & 392924.5 & 8.499 & 8.120 & 7.923 & B0.2IV & & 8 \\
\hline 125 & 203813.55 & 414416.3 & 9.865 & 8.635 & 7.952 & & & \\
\hline 128 & 202839.20 & 424628.1 & 8.883 & 8.392 & 7.997 & B0IIIe & AS 419 & 9 \\
\hline 136 & 202316.63 & 410925.0 & 10.106 & 8.937 & 8.120 & & & 5 \\
\hline 144 & 203845.88 & 420704.8 & 10.666 & 9.603 & 8.165 & & & 5 \\
\hline 148 & 203857.19 & 422240.9 & 13.160 & 10.307 & 8.214 & & & 5,10 \\
\hline 178 & 203041.44 & 394515.5 & 13.083 & 10.379 & 8.464 & & & 5,11 \\
\hline 180 & 202528.57 & 410826.1 & 12.325 & 10.207 & 8.464 & & & 5 \\
\hline 198 & 202559.59 & 401018.4 & 9.735 & 9.067 & 8.586 & & & 12 \\
\hline 199 & 203830.39 & 422826.4 & 10.198 & 9.291 & 8.599 & & & 5,13 \\
\hline 218 & 203812.88 & 405716.9 & 9.222 & 8.894 & 8.699 & B2V & & \\
\hline
\end{tabular}

Notes

1: Identified as a star with very strong $\mathrm{H} \alpha$ emission by Merrill \& Burwell (1950).

2: Wolf-Rayet star, infrared spectral type WC8; see Pasquali et al. (2002). It had been previously recognized as an emission-line star by Melikian \& Shevchenko (1990).

3: Already recognized as an emission line star by Dolidze (1975) (star 20-091).

4: In the W75N star forming region, strong excess at $8 \mu \mathrm{m}$ (Davis et al. 2007).

5: Suspected Herbig Ae/Be star basedon its infrared excess.

6: Double star, separation 1"1: type estimated as G3IV-V by Straizys et al. (1989) based on Vilnius photometry.

7: Identified as a star with weak $\mathrm{H} \alpha$ emission by Merrill \& Burwell (1950).

8: In DR 6.

9: Identified as a star with strong $\mathrm{H} \alpha$ emission by Merrill \& Burwell (1950).

10: In the HII region DR 21.

11: Possibly associated to IRAS $20288+3934$

12: Already recognized as an emission line star by Dolidze (1975) (star 20-089). In the HII region DR 5.

13: In DR 21.

Table 3. Lightly reddened OB stars.

\begin{tabular}{ccccccll}
\hline \hline Star & $\alpha(2000)$ & $\delta(2000)$ & $J$ & $H$ & $K_{\mathrm{S}}$ & Other IDs & Notes \\
\hline 27 & 202522.12 & 401301.2 & 6.836 & 6.623 & 6.495 & HD 194649 & 1 \\
30 & 203810.94 & 421026.0 & 7.047 & 6.670 & 6.556 & BD+41 3833 & 2 \\
31 & 202620.91 & 394010.3 & 7.105 & 6.802 & 6.580 & V455 Cyg & 3 \\
50 & 202555.09 & 412011.8 & 7.167 & 7.147 & 7.116 & HD 194779 & 4 \\
64 & 202830.24 & 420035.3 & 7.334 & 7.351 & 7.318 & HD 195229 & 5 \\
68 & 202310.46 & 404552.4 & 7.727 & 7.473 & 7.353 & BD +40 4146 & 6 \\
71 & 202736.77 & 420207.6 & 7.271 & 7.327 & 7.384 & HD 195089 & 7 \\
142 & 202244.75 & 404252.8 & 8.056 & 8.129 & 8.152 & HD 194194 & 8 \\
150 & 202743.62 & 403543.6 & 8.415 & 8.316 & 8.250 & BD +404179 & 9 \\
238 & 203828.89 & 400956.8 & 8.888 & 8.848 & 8.810 & BD +394263 & 10 \\
\hline
\end{tabular}

Notes

1: Possibly associated to DR 5. Classified as O6.5 by Morgan et al. (1955).

2: Classified as B8 by Straizys et al. (1989) based on Vilnius photometry.

3: Eclipsing binary (Giuricin et al. 1983), classified as B2p?e? by Morgan et al. (1955).

4: Classified as B3II by Morgan et al. (1955).

5: Classified as B0.2III by Walborn (1971).

6: In cluster NGC 6910; classified as B1 by Walker \& Hodge (1968).

7: Classified as B2IV by Guetter (1968).

8: In cluster NGC 6910; classified as spectral type B1.5IV by Walborn (1971).

9: Classified as O8V: by Morgan et al. (1955).

10: Ultraviolet-bright star, type B estimated by Carruthers \& Page (1984). 
Table 4. Foreground intermediate-type stars and lightly reddened, unclassified stars.

\begin{tabular}{cccccccc}
\hline \hline Star & $\alpha(2000)$ & $\delta(2000)$ & $J$ & $H$ & $K_{\mathrm{S}}$ & Other IDs & Notes \\
\hline \multicolumn{2}{c}{ Foreground stars } & & & & & & \\
28 & 202928.37 & 421900.3 & 6.814 & 6.579 & 6.521 & HD 195405 & 1 \\
35 & 203813.10 & 393249.5 & 6.941 & 6.705 & 6.660 & HD 196790 & 2 \\
57 & 202436.12 & 413002.8 & 7.603 & 7.281 & 7.192 & LTT 15972 & 3 \\
58 & 203729.72 & 423314.9 & 7.651 & 7.305 & 7.219 & HIP 101763 & 4 \\
69 & 202539.03 & 415345.6 & 7.673 & 7.428 & 7.370 & HD 229293 & 5 \\
73 & 202403.81 & 402022.7 & 7.653 & 7.454 & 7.391 & HD 229244 & 6 \\
85 & 202946.12 & 393901.1 & 7.842 & 7.572 & 7.516 & BD+39 4212 & 7 \\
92 & 203934.11 & 420130.3 & 7.784 & 7.670 & 7.616 & BD+41 3844 & 8 \\
174 & 202952.91 & 422127.4 & 8.714 & 8.481 & 8.423 & BD+41 3776 & 9 \\
203 & 204007.90 & 411510.8 & 9.067 & 8.708 & 8.615 & BD+40 4272 & 10
\end{tabular}

Lightly reddened stars with no classification in the visible

\begin{tabular}{lllllll}
139 & 203535.00 & 400129.5 & 8.380 & 8.211 & 8.128 & \\
191 & 203846.80 & 420106.4 & 8.908 & 8.615 & 8.552 & \\
197 & 202636.09 & 402829.1 & 8.810 & 8.606 & 8.583 & \\
201 & 202422.65 & 412608.5 & 8.901 & 8.652 & 8.612 & HD 229266 \\
210 & 202416.06 & 402926.6 & 8.964 & 8.686 & 8.642 & HD 229252 \\
214 & 203928.12 & 403615.1 & 9.020 & 8.747 & 8.677 & BD+40 4266 \\
216 & 202425.50 & 394928.4 & 8.831 & 8.748 & 8.695 & HD 229258 \\
231 & 203002.17 & 421434.1 & 9.053 & 8.845 & 8.767 & \\
234 & 202359.70 & 400746.8 & 8.952 & 8.820 & 8.783 & HD 229237 \\
239 & 203910.53 & 402006.4 & 9.149 & 8.861 & 8.813 & \\
249 & 202752.05 & 413120.0 & 9.143 & 8.895 & 8.847 & \\
\hline
\end{tabular}

Notes

1: Classified as G0Iab: (Nassau \& Morgan 1952) and as G2IV (Bidelman 1957).

2: Classified as F8IV (Nordstrøm et al. 2004).

3: High proper motion, horizontal branch star; see e.g. Behr (2003).

4: Close binary, high proper motion star; Fabricius \& Makarov (2000).

5: Classified as $\mathrm{K} 0$ in the HD catalog.

6: Classified as G0 in the HD catalog.

7: Classified as F8 in the SAO catalog.

8: Classified as F0V by Straizys et al. (1989) based on Vilnius photometry.

9: Classified as F5:V by Straizys et al. (1989) based on Vilnius photometry.

10: Nearby, high proper motion star at 75 pc according to the Hipparcos catalog. Suspected metal-poor subdwarf by Reid et al. (2001). 
F. Comerón et al.: The outskirts of Cygnus OB2, Online Material p 5

Table 5. Late B-F stars with Brackett absorption lines.

\begin{tabular}{|c|c|c|c|c|c|}
\hline Star & $\alpha(2000)$ & $\delta(2000)$ & $J$ & $H$ & $K_{\mathrm{S}}$ \\
\hline \multicolumn{6}{|c|}{ Unreddened or lightly reddened stars } \\
\hline 23 & 203933.32 & 403446.4 & 6.223 & 6.351 & 6.375 \\
\hline 26 & 202526.34 & 415437.4 & 6.603 & 6.573 & 6.487 \\
\hline 38 & 20263.85 & 40246.3 & 6.755 & 6.847 & 6.873 \\
\hline 41 & 203650.43 & 423630.1 & 7.203 & 7.04 & 6.986 \\
\hline 49 & 204053.3 & 41129. & 7.012 & 7.082 & 7.097 \\
\hline 53 & 202551.05 & 393753.0 & 7.540 & 7.231 & 7.153 \\
\hline 59 & 202643.77 & 392945.4 & 7.154 & 7.214 & 7.219 \\
\hline 77 & 203512.48 & 425115.3 & 7.765 & 7.595 & 7.457 \\
\hline 78 & 203126.34 & 395619.7 & 7.481 & 7.481 & 7.46 \\
\hline 83 & 203433.06 & 415947.9 & 7.532 & 7.542 & 7.499 \\
\hline 95 & 202651.15 & 415133.5 & 7.889 & 7.682 & 7.651 \\
\hline 99 & 202528.57 & 394736.7 & 7.625 & 7.703 & 7.698 \\
\hline 104 & 202842.56 & 392825.1 & 7.915 & 7.744 & 7.743 \\
\hline 106 & 202646.23 & 392744.1 & 7.719 & 7.749 & 7.778 \\
\hline 107 & 202515.68 & 405955.1 & 7.912 & 7.82 & 7.783 \\
\hline 126 & 202643.76 & 402050.1 & 7.848 & 7.932 & 7.967 \\
\hline 145 & 202321.83 & 413037.7 & 8.246 & 8.212 & 8.192 \\
\hline 147 & 203624.25 & 391140.7 & 8.207 & 8.191 & 8.213 \\
\hline 149 & 202923.57 & 40556.5 & 8.164 & 8.181 & 8.225 \\
\hline 153 & 202730.84 & 41261.2 & 8.329 & 8.276 & 8.265 \\
\hline 154 & 203159.74 & 42338.3 & 8.276 & 8.286 & 8.276 \\
\hline 155 & 203834.33 & 41937.4 & 8.469 & 8.28 & 8.279 \\
\hline 157 & 203244.44 & 391315.2 & 8.508 & 8.338 & 8.301 \\
\hline 161 & 204217.28 & 41294. & 8.947 & 8.512 & 8.336 \\
\hline 166 & 202713.68 & 423746.5 & 8.482 & 8.418 & 8.369 \\
\hline 171 & 204040.74 & 403743.8 & 8.602 & 8.428 & 8.409 \\
\hline 172 & 202544.55 & 40359.2 & 8.46 & 8.446 & 8.41 \\
\hline 173 & 20273.49 & 421757.1 & 8.383 & 8.402 & 8.414 \\
\hline 176 & 203243.5 & 391615.8 & 8.365 & 8.457 & 8.433 \\
\hline 188 & 202734.89 & 403221.9 & 8.838 & 8.605 & 8.54 \\
\hline 192 & 203346.89 & 395634.1 & 8.697 & 8.55 & 8.553 \\
\hline 195 & 203755.33 & 42751.1 & 8.794 & 8.633 & 8.579 \\
\hline 207 & 202551.15 & 403554.2 & 8.591 & 8.62 & 8.629 \\
\hline 208 & 20421.19 & 401442.8 & 8.701 & 8.665 & 8.633 \\
\hline 211 & 202515.1 & 4019.8 & 8.547 & 8.65 & 8.655 \\
\hline 212 & 203133.58 & 421214.2 & 8.558 & 8.635 & 8.66 \\
\hline 215 & 203722.33 & 392939 & 8.688 & 8.679 & 8.691 \\
\hline 217 & 202810.76 & 391247.8 & 8.746 & 8.758 & 8.696 \\
\hline 223 & 203734.28 & 394827.8 & 9.088 & 8.847 & 8.723 \\
\hline 226 & 204159.45 & 412658.9 & 8.865 & 8.787 & 8.737 \\
\hline 228 & 203634.73 & 41488.9 & 8.923 & 8.779 & 8.74 \\
\hline 233 & 202458.78 & 395021.7 & 8.713 & 8.776 & 8.776 \\
\hline 236 & 203938.95 & 403511 . & 8.733 & 8.812 & 8.803 \\
\hline 237 & 203336.33 & 394849.5 & 9.083 & 8.862 & 8.804 \\
\hline 241 & 202959.66 & 393426.6 & 8.901 & 8.858 & 8.822 \\
\hline 243 & $2028 \quad 27.27$ & 392228.4 & 8.824 & 8.843 & 8.824 \\
\hline 245 & $2032 \quad 14.14$ & 39440.1 & 8.927 & 8.85 & 8.825 \\
\hline 251 & 203034.23 & 391651.9 & 9.023 & 8.921 & 8.855 \\
\hline \multicolumn{6}{|c|}{ Reddened stars } \\
\hline 056 & 202858.75 & 401330.3 & 8.505 & 7.666 & 7.192 \\
\hline 109 & 203657.14 & 424210.0 & 9.688 & 8.442 & 7.788 \\
\hline 179 & 203119.00 & 42256.0 & 9.595 & 8.870 & 8.464 \\
\hline 240 & 203821.43 & 403039.7 & 10.548 & 9.416 & 8.815 \\
\hline
\end{tabular}


Table 6. Red giant candidates.

\begin{tabular}{ccccl}
\hline \hline Star & $\alpha(2000)$ & $\delta(2000)$ & $K_{\mathrm{S}}$ & Notes \\
\hline 1 & 202541.39 & 400033.5 & 4.186 & 1 \\
2 & 202741.05 & 395144.0 & 4.231 & \\
3 & 202417.99 & 411309.9 & 4.309 & \\
4 & 203518.04 & 415324.5 & 4.436 & 2 \\
9 & 203947.55 & 404704.1 & 4.885 & 3 \\
84 & 203040.10 & 422246.6 & 7.511 & \\
87 & 203640.68 & 400729.4 & 7.553 & \\
127 & 203100.20 & 422016.1 & 7.976 & \\
141 & 203922.70 & 411311.4 & 8.145 & \\
162 & 203353.54 & 425814.8 & 8.337 & \\
168 & 202239.72 & 403140.4 & 8.388 & \\
182 & 203528.30 & 425300.6 & 8.471 & \\
184 & 202422.85 & 395823.6 & 8.473 & \\
221 & 203846.36 & 422439.7 & 8.713 & 4 \\
225 & 204035.63 & 421017.0 & 8.736 & \\
247 & 204046.98 & 395005.4 & 8.839 & \\
248 & 203220.89 & 423249.1 & 8.845 & \\
\hline & & & &
\end{tabular}

Notes

1: HD 229291, classified in the visible as M0 (Neckel 1958).

2: HD 196360, classified in the visible as K0III (Bidelman 1957).

3: Classified as a carbon star by Chen \& Chen (2003); the $\mathrm{C}_{2} 1.77 \mu \mathrm{m}$ feature is barely visible in our near-infrared spectrum.

4: Possible Class I/II member of DR 21 (Davis et al. 2007).

Table 7. Carbon stars.

\begin{tabular}{cccc}
\hline \hline Star & $\alpha(2000)$ & $\delta(2000)$ & $K_{\mathrm{S}}$ \\
\hline 5 & 202310.08 & 405741.7 & 4.653 \\
6 & 204058.42 & 403347.8 & 4.676 \\
11 & 202256.90 & 411701.7 & 5.174 \\
24 & 204125.51 & 401521.8 & 6.413 \\
25 & 203547.30 & 424053.2 & 6.432 \\
43 & 203158.75 & 424808.0 & 7.014 \\
47 & 203610.71 & 391307.6 & 7.084 \\
52 & 202838.36 & 423547.7 & 7.150 \\
55 & 202436.14 & 405130.9 & 7.190 \\
76 & 203824.09 & 395152.3 & 7.453 \\
79 & 202726.95 & 402419.3 & 7.460 \\
90 & 204108.34 & 402414.2 & 7.567 \\
98 & 202626.64 & 402120.5 & 7.695 \\
102 & 203311.10 & 390224.3 & 7.731 \\
103 & 204118.63 & 402153.7 & 7.734 \\
105 & 202914.98 & 391923.5 & 7.750 \\
113 & 202528.25 & 414109.4 & 7.851 \\
114 & 203050.87 & 423814.4 & 7.852 \\
115 & 202704.60 & 423831.4 & 7.865 \\
117 & 202316.58 & 410744.0 & 7.876 \\
119 & 202727.82 & 424342.7 & 7.909 \\
123 & 202824.74 & 422010.2 & 7.924 \\
151 & 204126.61 & 414432.2 & 8.262 \\
165 & 203445.66 & 424913.3 & 8.359 \\
167 & 203857.69 & 410934.5 & 8.373 \\
187 & 203818.30 & 414239.5 & 8.529 \\
200 & 203305.99 & 390116.1 & 8.601 \\
213 & 202936.10 & 414924.9 & 8.674 \\
224 & 203937.54 & 421132.4 & 8.729 \\
\hline & & &
\end{tabular}


F. Comerón et al.: The outskirts of Cygnus OB2, Online Material $p 7$

Table 8. Likely long-period variables.

\begin{tabular}{ccccc}
\hline \hline Star & $\alpha(2000)$ & $\delta(2000)$ & $K_{\mathrm{S}}$ & Notes \\
\hline 8 & 203549.22 & 395956.7 & 4.801 & \\
40 & 203455.26 & 393635.2 & 6.977 & \\
51 & 203723.92 & 403033.0 & 7.129 & \\
54 & 203235.80 & 424235.4 & 7.178 & \\
61 & 202910.72 & 395059.3 & 7.284 & \\
80 & 202801.84 & 422314.0 & 7.483 & \\
89 & 202647.81 & 404647.6 & 7.563 & \\
91 & 203415.36 & 394459.8 & 7.603 & \\
111 & 202723.51 & 394201.6 & 7.832 & \\
121 & 202549.61 & 414012.6 & 7.917 & \\
124 & 203910.34 & 393349.0 & 7.931 & \\
160 & 202907.75 & 414912.5 & 8.328 & \\
163 & 202746.56 & 413202.4 & 8.345 & \\
177 & 204009.21 & 420117.5 & 8.441 & \\
\hline
\end{tabular}

Table 9. Extremely red objects.

\begin{tabular}{cccccccl}
\hline \hline Star & $\alpha(2000)$ & $\delta(2000)$ & $J$ & $H$ & $K_{\mathrm{S}}$ & IRAS source & Notes \\
\hline 158 & 202924.36 & 401115.6 & 12.325 & 11.082 & 8.320 & IRAS 20275+4001 & 1 \\
235 & 202644.43 & 400257.8 & 17.200 & 13.069 & 8.793 & IRAS 20249+3953 & 2 \\
\hline
\end{tabular}

Notes

1: Source AFGL 2591.

2: Possibly associated to DR 5. 